\title{
First body of evidence suggesting a role of a tankyrase- binding motif (TBM) of vinculin (VCL) in epithelial cells
}

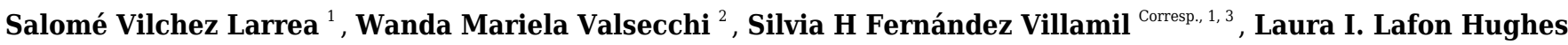 \\ Corresp. 4,5 \\ 1 Instituto de Investigaciones en Ingeniería Genética y Biología Molecular "Dr Héctor N. Torres", Consejo Nacional de Investigaciones Científicas y \\ Técnicas, Buenos Aires, Ciudad Autónoma de Buenos Aires, República Argentina \\ 2 Instituto de Química y Fisicoquímica Biológicas, Consejo Nacional de Investigaciones Científicas y Técnicas (CONICET), Buenos Aires, Ciudad Autónoma \\ de Buenos Aires, República Argentina \\ 3 Departamento de Química Biológica, Facultad de Farmacia y Bioquímica, Universidad de Buenos Aires, Buenos Aires, Ciudad Autónoma de Buenos Aires, \\ Argentina \\ 4 Grupo de Biofisicoquímica, Departamento de Ciencias Biológicas, Centro Universitario Regional Litoral Norte (CENUR), Universidad de la República, \\ Salto, Uruguay \\ 5 Departamento de Genética, Ministerio de Educación y Cultura, Instituto de Investigaciones Biológicas Clemente Estable, Montevideo, Uruguay \\ Corresponding Authors: Silvia H Fernández Villamil, Laura I. Lafon Hughes \\ Email address: silvia.villamil@gmail.com, lauralafon2010@gmail.com
}

Background. Adherens junctions (AJ) are involved in cancer, infections and neurodegeneration. Still, their composition has not been completely disclosed. Poly(ADPribose) polymerases (PARPs) catalyze the synthesis of poly(ADP-ribose) (PAR) as a posttranslational modification. Four PARPs synthesize PAR, namely PARP-1/2 and Tankyrase -1/2 (TNKS). In the epithelial belt, AJ are accompanied by a PAR belt and a subcortical F-actin ring. F-actin depolymerization alters the $A J$ and PAR belts while PARP inhibitors prevent the assembly of the AJ belt and cortical actin. We wondered which PARP synthesizes the belt and which is the PARylation target protein. Vinculin (VCL) participates in the anchorage of F-actin to the AJ, regulating its functions, and colocalized with the PAR belt. TNKS has been formerly involved in the assembly of epithelial cell junctions.

Hypothesis. TNKS poly(ADP-ribosylates) (PARylates) epithelial belt VCL, affecting its functions in $A J$ including cell shape maintenance. Materials and methods. Tankyrasebinding motif (TBM) sequences in hVCL gene were identified and VCL sequences from various vertebrates, $D$. melanogaster and $C$. elegans were aligned and compared. Plasma membrane-associated PAR was tested by immunocytofluorescence (ICF) and subcellular fractionation in Vero cells and TNKS role in this structure and cell junction assembly was evaluated using specific inhibitors. The identity of the PARylated proteins was tested by affinity precipitation with PAR-binding reagent followed by western blots. Finally, MCF-7 human breast cancer epithelial cells were subjected to transfection with Tol2-plasmids, carrying a dicistronic expression sequence including Gallus gallus wt VCL (Tol-2-GgVCL) or the same VCL gene with a point mutation in TBM-II (Tol2-GgVCL/*TBM) under the control of 
a $\beta$-actin promoter, plus green fluorescent protein following an internal ribosome entry site (IRES-GFP) to allow the identification of transfected cells without modifying the transfected protein of interest. Results and discussion. In this work, some of the hypothesis predictions have been tested. We have demonstrated that: (1) VCL TBMs were conserved in vertebrate evolution while absent in C. elegans;(2) TNKS inhibitors disrupted the PAR belt synthesis, while PAR and an endogenous TNKS pool were associated to the plasma membrane; (3) a VCL pool was covalently PARylated; (4) transfection of MCF-7 cells leading to overexpression of Gg-VCL/*TBM induced mesenchymal-like cell shape changes. This last point deserves further investigation, bypassing the limits of our transient transfection and overexpression system. In fact, a $5^{\text {th }}$ testable prediction would be that a single point mutation in VCL TBM-II under endogenous expression control would induce an epithelial to mesenchymal transition (EMT). To check this, a CRISPR/Cas9 substitution approach followed by migration, invasion, gene expression and chemo-resistance assays should be performed. 


\section{First body of evidence suggesting a role of a}

\section{Tankyrase-Binding Motif (TBM) of Vinculin (VCL) in}

\section{3 epithelial cells.}

4

7

8

Salomé Vilchez Larrea ${ }^{1}$, Wanda M. Valsecchi ${ }^{2}$, Silvia Fernández Villamil 1,3凶 , Laura LafonHughes $4,5 \square$

${ }^{1}$ Instituto de Investigaciones en Ingeniería Genética y Biología Molecular "Dr. Héctor N. Torres" Consejo Nacional de Investigaciones Científicas y Técnicas, Ciudad Autónoma de Buenos Aires, Argentina (INGEBI-CONICET) ${ }^{2}$ Instituto de Química y Fisicoquímica Biológicas (IQUIFIB-CONICET)

${ }^{3}$ Departamento de Química Biológica, Facultad de Farmacia y Bioquímica, Universidad de Buenos Aires, Ciudad Autónoma de Buenos Aires, Argentina

${ }^{4}$ Departamento de Genética, Instituto de Investigaciones Biológicas Clemente Estable (IIBCE), Montevideo, Uruguay

${ }^{5}$ Grupo de Biofisicoquímica, Departamento de Ciencias Biológicas, Centro Universitario Regional Litoral Norte, Universidad de la República (CENUR-UdelaR), Salto, Uruguay هCorresponding Authors: Laura Lafon-Hughes ${ }^{4,5}$ Avenida Italia 3318, Montevideo, 11600, Uruguay Email address: lauralafon2010@gmail.com

Silvia H. Fernández Villamil ${ }^{1,3}$ Vuelta de Obligado 2490, Buenos Aires, C1428 ADN, Argentina Email address: s.villamil@ingebi.conicet.gov.ar

\section{Abstract}

Background. Adherens junctions (AJ) are involved in cancer, infections and neurodegeneration. Still, their composition has not been completely disclosed. Poly(ADP-ribose) polymerases (PARPs) catalyze the synthesis of poly(ADP-ribose) (PAR) as a posttranslational modification. Four PARPs synthesize PAR, namely PARP-1/2 and Tankyrase -1/2 (TNKS). In the epithelial belt, AJ are accompanied by a PAR belt and a subcortical F-actin ring. F-actin depolymerization alters the AJ and PAR belts while PARP inhibitors prevent the assembly of the AJ belt and 
33

34

35

36

37

38

39

40

41

42

43

44

45

46

47

48

49

50

51

52

53

54

55

56

57

58

59

60

61

62 63

64

65 66

cortical actin. We wondered which PARP synthesizes the belt and which is the PARylation target protein. Vinculin (VCL) participates in the anchorage of F-actin to the AJ, regulating its functions, and colocalized with the PAR belt. TNKS has been formerly involved in the assembly of epithelial cell junctions.

Hypothesis. TNKS poly(ADP-ribosylates) (PARylates) epithelial belt VCL, affecting its functions in AJ including cell shape maintenance.

Materials and methods. Tankyrase-binding motif (TBM) sequences in hVCL gene were identified and VCL sequences from various vertebrates, D. melanogaster and C. elegans were aligned and compared. Plasma membrane-associated PAR was tested by immunocytofluorescence (ICF) and subcellular fractionation in Vero cells and TNKS role in this structure and cell junction assembly was evaluated using specific inhibitors. The identity of the PARylated proteins was tested by affinity precipitation with PAR-binding reagent followed by western blots. Finally, MCF-7 human breast cancer epithelial cells were subjected to transfection with Tol2-plasmids, carrying a dicistronic expression sequence including Gallus gallus wt VCL (Tol-2-GgVCL) or the same VCL gene with a point mutation in TBM-II (Tol2-GgVCL/*TBM) under the control of a $\beta$-actin promoter, plus green fluorescent protein following an internal ribosome entry site (IRES-GFP) to allow the identification of transfected cells without modifying the transfected protein of interest.

Results and discussion. In this work, some of the hypothesis predictions have been tested. We have demonstrated that: (1) VCL TBMs were conserved in vertebrate evolution while absent in C. elegans; (2) TNKS inhibitors disrupted the PAR belt synthesis, while PAR and an endogenous TNKS pool were associated to the plasma membrane; (3) a VCL pool was covalently PARylated; (4) transfection of MCF-7 cells leading to overexpression of Gg-VCL/*TBM induced mesenchymal-like cell shape changes. This last point deserves further investigation, bypassing the limits of our transient transfection and overexpression system. In fact, a $5^{\text {th }}$ testable prediction would be that a single point mutation in VCL TBM-II under endogenous expression control would induce an epithelial to mesenchymal transition (EMT). To check this, a CRISPR/Cas9 substitution approach followed by migration, invasion, gene expression and chemo-resistance assays should be performed.

KEYWORDS: poly(ADP)ribose, PARP, tankyrase, Vinculin, adherens junctions, Vero, MCF-7, EMT

\section{Introduction}

Epithelial morphology, tensile/traction resistance and polarization maintenance rely on intercellular and cell-matrix junctions, where cytoskeleton fibers are anchored. Apart from having structural functions, cell junctions or its molecular components take part in intracellular 
68

69 70

71

72 73

74 75 76

and intercellular cell signaling nets. An increasing number of Nuclear and Adhesion Complex proteins (NACos) can alternatively be part of the cell junction, or be translocated to the nucleus to work as pro-cell cycling transcription factors, acting as a switch to coordinate cell rounding in mitosis $v s$. cell anchoring during differentiation [1,2]. The adherens junctions epithelial belt -or zonula adherens- allows the anchorage of the subcortical actin ring. Although the complete molecular composition of adherens junctions has been evasive and some authors have suggested the existence of still-not discovered components [3-5]. E-cadherin, $\alpha$-catenin and $\beta$-catenin have been identified as adherens junctions core proteins. In turn, Vinculin (VCL) links the core proteins to the subcortical actin cytoskeleton. While $\beta$-catenin is a well-studied NACo $[1,2]$, nuclear E-cadherin has been detected in lung cancer cells [6]. Interestingly, the adherens junctions epithelial belt is disassembled during a process that facilitates cancer progression which is called Epithelial to Mesenchymal Transition (EMT). EMT involves coordinated changes in cell shape and adhesion, epithelial polarization loss, molecular markers alterations, improved migration and invasion capacity and increased chemoresistance [7-9].

Poly(ADP-ribose) or PAR is a polymer synthetized by poly(ADP-ribose) polymerases (PARPs) from nicotinamide adenine dinucleotide $\left(\mathrm{NAD}^{+}\right)$, as a posttranslational protein modification. PAR can be lineal or ramified, comprising up to 400 residues. PAR is degraded by poly(ADPribose) glycohydrolase (PARG) or other enzymes [10-13]. As PAR is rich in phosphates and is negatively charged like nucleic acids, it acts as a "glue" to stabilize protein complexes. Interestingly, specific protein domains act as "readers", recognizing PAR substructures. The Macrodomain identifies terminal ADP-ribose groups, the WWE domain binds iso-ADP-ribose and the PBZ domain recognizes adjacent ADP-ribose groups. In contrast, long and even branched PAR is preferred by RNA recognition motif (RRM) or PAR-binding motif (PBM) $[13,14]$. In Vero renal epithelial cells, there is a PAR belt associated to the adherens junctions belt that has been evidenced by immunocytofluorescence (ICF) with anti-PAR antibodies. Such PAR belt colocalizes with VCL [15]. Successive works in the past three decades have implied the VCL pools bound to adherens junctions or focal contacts in the regulation of epithelial cell polarity, adhesion, migration, invasion, and cycling as well as death resistance [16-27]. Recent nuclear VCL detection [28,29] indicates that it may behave as a NACo. Cytochalasin D induces the disassembly of the cortical actin ring together with the disruption of the PAR belt. Conversely, if the PARP inhibitor XAV939 is applied at the moment of cell seeding, PAR belt 
99 synthesis, cell junction formation and cell adhesion are hampered [15]. Interestingly, during the 100 EMT, the PAR belt is disassembled together with the adherens junctions epithelial belt [30].

101 Although the PARP family comprises 18 members in humans [31,32], only four "true-PARPs" 102 synthetize PAR: PARP-1, PARP-2, Tankyrase-1 and Tankyrase-2 (TNKS-1 \& TNKS-2). PARP-

103 1, the canonical family member, is mainly nuclear and has been extensively involved in 104 chromatin remodeling, imprinting and DNA repair [33-35], functions that display partial 105 redundancy with those attributed to PARP-2. TNKS can be localized in cell membranes or 106 associated to telomeres and the mitotic spindle proteins [35-39]. Interestingly, in MDCK renal 107 epithelial cells, overexpressed TNKS-1 is recruited from the cytoplasm to the lateral plasma 108 membrane upon formation of E-cadherin-based cell-cell contacts while it is displaced by 109 extracellular calcium chelation that prevents intercellular adhesion [40], demonstrating its 110 involvement in cell junction dynamics. A role of TNKS in the maintenance of tight junctions and 111 epithelial polarization has been independently demonstrated [41]. TNKS recognizes its substrates 112 through the binding of its ankyrin-repeated clusters (ARC) domains to one or more Tankyrase 113 Binding Motifs (TBMs). TNKS binding via TBM is necessary but not sufficient to achieve 114 substrate poly(ADP-ribosylation) or PARylation. Although TBMs were initially described as 115 octamers, the most accepted current view is that a canonical TBM (c-TBM) involves at least a 116 hexamer $\underline{\mathbf{R X X O X}} \underline{\mathbf{G}}$, where X is any aminoacid and $\mathrm{O}$ is Gly, Pro, Ala or Cys. The existence of 117 non-canonical TBMs (nc-TBMs) or indirect interactions with some PARylation substrates was 118 postulated to explain the fact that in certain TNKS KO cells, there is an increase of many 119 proteins which do not contain a c-TBM (presumably due to lack of PARylation/degradation) 120 [39]. Non-canonical motifs (nc-TBMs) were later described as heptamers $\underline{\mathbf{R} X X X \mathbf{O X G}}$ [39,42$12145]$.

122

123 Here, we challenged the hypothesis that TNKS is involved in the synthesis of the PAR belt, with 124 VCL being one of its PARylation targets. More interesting, we investigated if some VCL 125 functions, like subcortical actin ring anchorage and epithelial cell shape maintenance, depend on 126 its PARylation by TNKS. Sequence analysis disclosed that vertebrate VCL harbors 3 TBMs, 127 which we identified as c-TBM-I, c-TBM-II (448-453) and nc-TBM-III. The plasma membrane 128 subcellular fraction (PMF) from Vero cells harbored PAR and TNKS and a VCL pool was a 129 PARylation target. TNKS inhibitors disrupted the epithelial belt. Overexpression of VCL with 
130 the cTBM-II mutated induced mesenchymal-like cell shape changes in MCF-7 cells. These 131 results provide the first pieces of evidence in favor of our hypothesis and suggest a role for 132 Tankyrase-Binding Motif (TBM) of Vinculin (VCL) in epithelial cells.

\section{Materials and methods}

134 1. Cell culture

135 Vero cells (African green monkey kidney cells, ATCC CCL-81), were routinely cultured in 136 MEM (PAA E15-888 or Capricorn MEM-STA) supplemented with $10 \%$ fetal bovine serum 137 (FBS; PAA A15-151 or Capricorn), $100 \mathrm{U} / \mathrm{mL}$ penicillin and $100 \mu \mathrm{g} / \mathrm{mL}$ streptomycin (Sigma138 Aldrich) at $37^{\circ} \mathrm{C}$ and $5 \% \mathrm{CO}_{2}$. ATCC MCF-7 cells were received in passage number $6(\mathrm{P} 6)$ as a 139 kind gift from Archana Dhasarathy and Sergei Nechaev, University of North Dakota, USA. 140 MCF-7 cells were cultured up to passage 14 (P14) in Capricorn DMEM-HPSTA with 10 \% FBS $141(\mathrm{GIBCO})$ in an incubator at $37^{\circ} \mathrm{C}$ and $5 \% \mathrm{CO}_{2}$ or backed up frozen in $5 \%$ dimethylsulfoxide 142 (DMSO) and $20 \%$ FBS in DMEM-HPSTA.

\section{2. Protein extraction from subcellular fractions}

144 Subcellular fractions were obtained from control cultures. The plasma membrane protein 145 extraction kit (101Bio, \#P503) was used according to the manufacturer's instructions in order to 146 extract proteins from nuclear fraction (NF), cytoplasmic fraction (CF), internal membrane 147 fraction (IMF) and plasma membrane fraction (PMF) of Vero cells (about $3 \times 10^{7}$ cells). The 148 fractions were then subjected to western blot to detect TNKS and PAR. Fraction controls 149 included Lamin A/C (NF), CALR3 (CF), GRASP (IMF) and PMCA (PMF).

\section{3. Whole cell protein extraction and affinity precipitation of PARylated proteins}

151 Briefly, cells were grown in a T75 flask, washed, harvested by scraping and resuspended in a 152 lysis buffer (50 mM Tris, $\mathrm{pH}$ 8, $200 \mathrm{mM} \mathrm{NaCl}, 1 \mathrm{mM}$ EDTA, 1\% Triton X-100, 10\% glycerol, 1 153 $\mathrm{mM}$ DTT, $0.1 \% \mathrm{SDS}$, and protease inhibitors $)$. The extract was clarified $\left(10 \mathrm{~min}, 15000 \mathrm{~g}, 4^{\circ} \mathrm{C}\right)$, and then subjected to affinity precipitation of PARylated proteins using WWE affinity resin

155 (Tulip \#2334) or Af1521 Macrodomain affinity resin (Tulip \#2302), according to the manufacturer's instructions. The resin was equilibrated in lysis buffer $(10000 \mathrm{~g}, 20 \mathrm{sec})$ and total protein extract was added to the resin pellet and incubated $\mathrm{ON}$ in a cold chamber on a rotary shaker. The following day, the resin with the bound PARylated proteins was precipitated by 
159 centrifugation and the supernatant was saved as "flowthrough" (FT). The resin pellet was washed 160 three times in lysis buffer. Affinity precipitated proteins (AP) were eluted by incubating in $50 \mu \mathrm{L}$ 161 protein loading buffer at $99^{\circ} \mathrm{C} 10 \mathrm{~min}$.

162 4. SDS-PAGE and western blot

163 Protein concentration was estimated by Bradford when appropriate. SDS-PAGE was done as 164 described by Laemmli (1970). Semi-dry protein transfer onto nitrocellulose membranes was 165 achieved using BiometraFastBlot (Analytik Jena). Membranes were blocked with 5\% non-fat 166 milk suspension in $0.05 \%$ TBS-Tween for $1 \mathrm{~h}$ and then incubated with the primary antibody ON 167 and secondary antibody for $1 \mathrm{~h}$. Western blots images were taken using the GeneGnome XRQ 168 Chemiluminescence imaging system after developing with Western Lightning Plus ECL 169 Substrate (Perkin Elmer). When necessary, membrane stripping was achieved by incubation in a 170 Mild Stripping buffer (1.5\% Glycine, 0.1\% SDS, 0.1\% Tween-20, pH 2.2). The antibodies used 171 and their correspondent concentrations are listed in Table 1.

5. Cell seeding in the presence of Tankyrase inhibitors to monitor epithelial belt 173 assembly.

174 TNKS inhibitors were kindly provided by Dr. Lari Lehtiö (Biocenter Oulu, Faculty of

175 Biochemistry and Molecular Medicine, University of Oulu, Finland). The reported potencies and 176 IC50 are summarized in Table 2 [46-52]. Vero cells were treated with TNKS inhibitors (TNKSi) 177 at the moment of seeding. Their effect on the PAR belt and on the accompanying cortical actin 178 ring was evaluated qualitatively by immunocytofluorescence $5 \mathrm{~h}$ after seeding. Alternatively, 179 less cells were seeded and TNKSi was added on the monolayer and cells were fixed after 180 reaching confluency.

181

182 6. Indirect immunocytofluorescence.

183 Indirect immunocytofluorescence was carried as in Lafon-Hughes et al. (2014) on $12 \mathrm{~mm}$ round 184 cover glasses placed in 24-well plates. Briefly, cells were fixed in 4\% paraformaldehyde (PFA), 185 permeabilized and subjected to blocking. Then, they were incubated with specific primary 186 antibodies (see Table 1$)$ diluted in blocking buffer ( $0.2 \%$ Tween, $1 \%$ BSA in fPBS) for $2 \mathrm{~h}$ at 37 $187{ }^{\circ} \mathrm{C}$. After washing, cells were incubated (1h, RT) with the correspondent anti-antibody mix in 188 blocking buffer and/or cytopainter (ab176756). After washing, nuclei were counterstained with 
189 DAPI, coverslips were mounted in Vectashield (Vector 94010) or Prolong Gold (Molecular 190 Probes P36930) and sealed. In order to check the specificity of the signals, control without 191 primary antibodies were carried in parallel.

$1927 . \quad$ Epifluorescence and confocal microscopy.

193 Images were recorded with an epifluorescence microscope (40x objective) with DP controller 194 software, an Olympus BX61/ FV300 (Tokyo, Japan) with a Plan Apo 60 x/1.42 NA oil 195 immersion objective or a ZEISS LSM800-Airyscan (Oberkochen, Germany) confocal 196 microscope. Original images were taken in the same conditions as reference images of controls 197 without primary antibodies at the same microscopy session. All images in each experimental 198 series were taken with the same setting at the same confocal session. If modified, all were 199 subjected to the same degree of brightness/contrast adjustment and Gaussian blur filtering, 200 including the control without a primary antibody. The ImageJ free software was used for image 201 processing.

202 8. VCL vs PAR colocalization in epithelial belts: overlapping pixels highlight and 203 quantification examples.

204 Colocalization can be analyzed in terms of signals overlap or signals intensity correlation.

205 8.1. Overlapping Pixels Highlight. Colocalization was initially evidenced qualitatively by 206 visual inspection. As PAR signal was usually lower than VCL signal, the signal overlap 207 visualization was facilitated through the creation of masks. Masks creation involves the selection 208 of an intensity threshold above which there is a real signal and below which there is noise. The 209 same threshold was used in controls without primary antibody (obtaining empty masks) as an 210 extreme caution to avoid taking noise as signal. As masks are segmented images relying on 211 values of 1 or 0 in each pixel, the multiplication of masks images (using Image J/Image 212 Calculator) allowed a perfect outline of the overlapped regions (in yellow).

213 8.2. Exemplar Overlapping Extent Quantification. Mander's indexes M1 and M2 calculated

214 using masks reflected the overlapping extent: the proportion of positive pixels in channel A that 215 also had a positive signal in channel B or vice versa (the proportion of positive pixels in channel 216 B that harbored a positive signal in channel A).

217 8.3. Exemplar Intensity Correlation Analysis. Pearson Coefficient (Rr), which ranges from -1 to $218+1$ (from anti-colocalization to complete colocalization) and Li's ICQ (which ranges from -0.5 to 
$219+0.5$ ) were calculated on ROIs (regions of interest). PDMs (products of the differences from the 220 means) were localized. PDMs can be double positives (both above the mean), represented as $221++$ PDMs, or double negatives (both below the mean). ++PDMs were mapped on the image (in 222 violet).

223 8.4. Quantitative measures by FRET on indirect ICF images (see Supplemental Article 1) 224

9225 226 227

228

229

230

231

232

233

234

235

236

237

238

239

240

241

242

243

244 245

Relative expression of VCL in cells transfected with Tol2-GgVCL vs Tol2-VCL/*TBM. Intensity quantification on confocal images was used to estimate the relative expression of VCL and GFP in transfected cells. Whole-cell contours and nuclear contours were used as ROIs to determine whole-cell GFP, whole-cell VCL, nuclear GFP and cytoplasmic VCL.

\section{Cell Shape index: combined Roundness and Circularity.}

Epithelial cells display tension in their adherens junctions belts and subcortical actin rings, giving rise to straight lines. Besides, even in the absence of neighbor cells, they have quite sharp borders. In contrast, mesenchymal or mesenchymal-like cells have serrated, wavy junctions and an irregular outline due to the presence of filopodia and lamellipodia (which can be decorated with ruffles) [53]. To be able to quantify and compare morphological changes, it was required to express these morphologies in terms of numbers. Image J offers measurements of Roundness and Circularity. While Roundness accounts for gross changes (from circular to ovoid or triangular), it is insensitive to straight vs serrated or undulated borders. On the other hand, Circularity is very sensitive to such edge characteristics. Thus, we defined a Cell Shape Index ( CShin) combining Roundness and Circularity.

\section{CShin $=\frac{\text { Roundness }}{\text { Circularity }}$}

Figure 1A depicts values of CShin associated to some geometric figures (on the left). CShin is sensitive to radial symmetry, being higher for a circle than for an ellipse or higher for a square than a rectangle. If the clean edge of each geometric form is substituted by a serrated or undulated border, CShin values show a sharp increase. As can be seen on Fig. 1 A (right), CShin is very sensitive to the edge characteristics of the shape. For example, CShin $=1$ for a perfect 
246 circle and CShin $=1.81$ for a form that conserves the circular proportions but has an undulated 247 edge.

248 As a proof of concept, CShin was applied to a well-characterized cell model of an epithelial to 249 mesenchymal transition (EMT). NMuMG (NAMRU Murine Mammary Gland) cells were 250 measured untreated (when they have epithelial characteristics) or after a treatment with TGF- $\beta$ 251 (when they have mesenchymal characteristics).

252 The Plotly Chart Studio online graph maker (https://chart-studio.plotly.com/create/box-plot/\#/) 253 was used to build box \& whisker graphs plus represent all the individual data points. Statistics 254 were done using the free software available online at astatsa.com, attributed to Navendu 255 Vasavada (2016). As CShin statistical distribution is not normal, medians were compared using 256 Mann-Whitney non-parametric test. Kruskall-Wallis non-parametric ANOVA followed by 257 Conover test adjusted by Benjamini-Hochberg FDR method.

258 The shape change was evidenced as an increased CShin index (Fig. 1 B). In this model, CShin 259 mean and median were below 1 for epithelial cells and above 1 for mesenchymal cells, with a 260 significant difference $\left(\mathrm{p}=2.413887 \times 10^{-8}\right)$. Thus, the index proved to be useful to evidence the 261 cell shape change from an epithelial to a mesenchymal cell.

\section{11. TBM analysis in $\mathrm{hVCL}$ and homologues}

263 Using SnapGene viewer, hVCL protein RefSeq sequence was searched for the c-TBM,

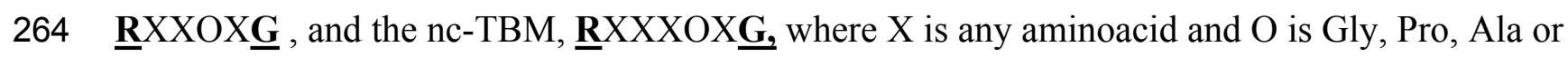
265 Cys. After global lineal-sequence alignment was made using ClustalX 2.0 for Windows [54], 266 TBM conservation was checked among VCL homologs in different representative species. The 267 Reference Sequences used were (as in Han et al 2017, [55]): Human NP_003364.1, Mouse 268 NP_033528.3, Chicken NP_990772.1, Xenopus NP_001090722.1, Zebrafish NP_001122153.1, 269 Drosophila NP-476820.1 and C elegans NP_501104.2). BLOSUM series matrix. Comparison 270 between hVCL and dVCL sequences were made using ExPASy alignment tool, SIM [56]. Result 271 indicated as Drosophila 1 corresponds to comparison matrix PAM400, while result indicated as 272 Drosophila 2 corresponds to comparison matrix BLOSUM30, using equal parameters in both 273 cases. 
274 Drosophila VCL structure was predicted using CPHmodels-3.0 [57]. Structural alignment and 275 Figures were made using YASARA [58].

276 12. Generation of eukaryotic expression vectors with WT-GgVCL (Tol2-GgVCL/WT) or 277 G454V-GgVCL (Tol2-GgVCL/*TBM).

278 To disrupt the association of VCL with TNKS, Gly454 (in TBM-II) was substituted for a Val 279 residue. A ggc codon was substituted for gta by directed/circular mutagenesis using custom 280 primers G454V (Table 3) and following the protocol published by Wang and Wilkinson [59]. $281 \mathrm{pET15b/GgVcl} \mathrm{1-1066} \mathrm{(Addgene} \mathrm{\# 46171)} \mathrm{bearing} \mathrm{the} \mathrm{complete} \mathrm{Gallus} \mathrm{gallus} \mathrm{VCL} \mathrm{sequence}$ 282 was used as template. An AccI (BioLabs R0161S) (gtagac) site generated by the point mutations 283 introduced was used as diagnostic recognition site. The new plasmid, $\mathrm{pET} 15 / \mathrm{GgVcl} /{ }^{*} \mathrm{TBM}$, will 284 soon be available in Addgene (\#162781).

285 The GgVCL and GgVCL/*TBM ORFs were then cloned into TOPO TA \#450640 through the 286 topo cloning reaction, following the manufacturer's instructions, an then into a middle entry 287 plasmid with multiple cloning sites (Tol2 Kit\#237, pME-MCS), to obtain the pME-VCL vector.

288 The final mammalian expression vector was obtained through the use of Tol2 kit LR [60][61]. 289 The LR reaction involved the use of Gateway LR Clonase II Plus Enzyme Mix (Invitrogen 290 12538-120) to fuse 4 plasmids into a single final vector: the 5 'entry plasmid with a $\beta$-actin 291 promoter called p5E-actin (\#299), the middle entry plasmid with the VCL insert pME-VCL (wt 292 or mut), the 3'entry plasmid p3E-IRES-nls-GFP (\#391) and the destination vector pDestTol2pA2 293 (\#394). The resulting 14.5 KB eukaryotic expression vectors carried the WT or mutant VCL 294 under a $\beta$-actin promoter and a GFP coding sequence driven by an internal ribosome entry site 295 (IRES-GFP). Tol-2 GgVCL and Tol-2 GgVCL/*TBM (that have been deposited in a repository 296 (Addgene \#162787 and \#162790) constructs were amplified in NEB C3019I bacteria and 297 corroborated by primer walking sequencing (Macrogen).

13. Transfection of MCF- 7 cells with Tol2-GgVCL or Tol2-GgVCL/*TBM

299 FUGENE transfection reaction: DNA (3:1) was used to transfect MCF-7 undisrupted 300 monolayers. Cells were monitored under epifluorescence and phase contrast or subjected to ICF 301 at the indicated times. $1 \mu \mathrm{g}$ Pmax GFP plasmid ( $\sim \mathrm{KB})$ from Amaxa Mouse/Rat Hepatocyte 302 Nucleofector kit was used as a positive control to evaluate transfection efficiency of a DNA 303 plasmid. 


\section{Results}

305 1. VCL sequence harbors three TBMs conserved in vertebrates.

306 All confirmed TNKS substrates to date contain one or more TBMs that are recognized via TNKS

307 ARC domains (ARC1, ARC2, ARC4 and ARC5) [43]. Therefore, proteins harboring a TBM are

308 putative TNKS PARylation targets. We examined the presence of TBMs in hVCL. Also, a

309 comparative alignment was done with VCL sequences from model organisms including some

310 vertebrates and two invertebrates: Drosophila melanogaster, whose adherens junctions are quite

311 similar to those of vertebrates, and Caenorhabditis elegans, whose adherens junction proteins

312 are not essential for general cell adhesion or for epithelial cell polarization.

313 Human VCL has three TBMs (Fig. 2 A): TBM-I is $\underline{\text { RARGQG }}$ at positions 339-344 (exons 8-9),

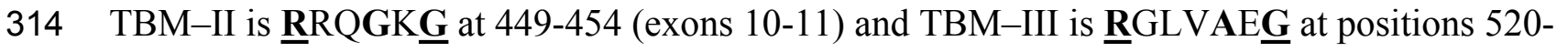

315526 (exon 12). The first two sites are canonical ( $\underline{\mathbf{R X X O X}} \underline{\mathbf{G}}$ ) and correspond to peripheral, easily

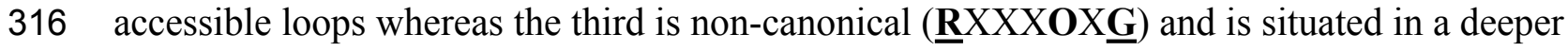
317 position, on a helix.

318 All TBMs were conserved in other vertebrates such as Mus musculus, Gallus gallus, Xenopus 319 laevis and zebrafish, while only one of them was also found in Drosophila melanogaster. In 320 contrast, none of them was present in Caenorhabditis elegans (Fig. 2A). It is interesting to note 321 that the single TBM domain of Drosophila could align with both canonical TBMs (TBM I and 322 II) of hVCL. Hereafter structural comparison between hVCL and dVCL was done. The 323 superimposition of hVCL with the predicted 3D-structure of dVCL indicated that Drosophila 324 TBM was located in the same relative position in the structure than the human VCL TBM-II 325 (Fig. 2 B).

2. Endogenous PAR and TNKS were detected in the plasma membrane fraction of Vero cells

328 PAR had been previously detected associated to the epithelial belt in Vero cells by ICF using an 329 anti-PAR antibody [15]. Now we corroborated PAR presence in subcellular fractions whose 330 purity was checked with specific markers (Fig. 3 A). Instead of an antibody, the novel anti-PAR 331 reagent MABE 1031 was used to evidence the presence of PAR associated to the plasma membrane fraction (PMF). MABE1031 (AB_2665467) is a recombinant protein comprising the 
333 WWE domain from RNF146 and the Fe region of rabbit IgG. The latter is recognized by anti334 rabbit secondary antibodies. Thus, it can be handled as an equivalent to a primary antibody in 335 ICF, WB or affinity pull-down applications.

336 TNKS presence in the internal membrane fraction (IMF) was conspicuous and a slighter signal 337 was also detected in nuclear fraction (NF), cytoplasmic fraction (CF) and plasma membrane 338 fraction (PMF) (Fig. 3 A). The cell fractionation results matched with ICF images (Fig. 3 B - E). 339 In ICF, a TNKS 'hat' can be observed on one side of the nucleus, presumably corresponding to 340 the Golgi complex. It is possible to see that a mild/slight TNKS signal spreads towards the rest of 341 the cell, reaching the plasma membrane or subcortical region sometimes (Fig. 3 D, E). This

342 TNKS distribution was robust, since it was observed in ICF experiments carried using two 343 different anti-TNKS antibodies designed to recognize the $\mathrm{Nt}$ or the $(\mathrm{Ct})$ catalytic domain, as well 344 as under two different fixation protocols (Fig. S 1).

345

346 3. TNKS inhibitors disrupted the PAR belt synthesis and altered cell shape.

347 A tight adherens junctions belt, indicative of tension, involves a straight PAR belt and a straight

348 subcortical actin belt. In contrast, an incomplete lax belt is associated with punctuated (less

349 abundant) or serrated PAR and F-actin signals.

350 Four TNKS inhibitors with different potency and specificity towards TNKS-1 and TNKS-2

351 (Table 2), were added to cell cultures at the moment of seeding (Fig. 4). Results were ordered 352 according to TNKSi in vitro specificity, starting with TNKS-1-prone FLALL( Fig. 4 B, G, L), 353 then MN64, G007LK and in the end the TNKS-2 selective OD35 (Fig. 4 E, J, O). All of them 354 hindered PAR belt synthesis. A punctuated or zipper-like discontinuous distribution of PAR was 355 observed (Fig. 4 B - O). The PAR belt did not completely disappear, which could be explained 356 by incomplete TNKS inhibition. Cortical F-actin changed accordingly (Fig. $4 \mathbf{G}-\mathbf{J}$ ), reinforcing 357 our previous results correlating PAR and F-actin distributions in epithelial cells and nerves $358[15,62]$. In the presence of MN64, less cells remained attached fixation, after the PBS wash done 359 previous to fixation. Such a cell density decrease has been previously documented in the 360 presence of a TNKS and PARP-1/2/3 inhibitor called XAV939 [15]. 
361 With the aim of quantifying changes in cell shape, particularly those involving cell contours, we

362 designed a Cell Shape Index or CShin (see Materials and Methods, section 9, Fig. 1).

363 Median CShin (Fig. 5) was below 1 in control cells, as expected for epithelial cells according to

364 the index validation in NMuMG cells (Fig. 1 B). No effect of the PARP-1/2/3 inhibitor Olaparib

365 (OLA) was detected. In contrast, all TNKSi, namely FLALL, MN64, G007LK and OD35,

366 induced a significative increase of CShin indicating a measurable change in cell contours.

367 We did not find a correlation among TNKSi selectivity and the observed effects on PAR belt, the 368 subcortical F-actin cytoskeleton and CShin. The potency and specificity of the inhibitors is 369 determined in vitro while, in whole cells, factors as permeability, subcellular distribution, 370 stability and metabolic alterations may affect the effective dose and outcome. Therefore, a 371 straight-forward correlation was not necessarily expected.

372

373 As stated in the introduction, in epithelial cells the subcortical actin ring and the PAR belt are 374 somehow associated. We are now arguing that PARylation of a target protein joining adherens 375 junctions and the F-actin cytoskeleton by TNKS1/2 is at least one of the factors that could 376 explain this association.

377 4. A VCL pool was a PARylation target.

378 Using a WWE-affinity resin, covalently PARylated proteins were affinity precipitated. Next, cell 379 junction or cytoskeletal proteins were tested by WB as putative PARylation targets (Fig. 6 A). 380 Interestingly, while $\beta$-catenin, E-cadherin, actin and $\alpha$-tubulin were recovered only in the flow 381 through (FT), a fraction of VCL was affinity precipitated (AP) by the WWE-affinity resin which 382 consists of highly purified GST-RNF146(100-175) fusion protein bound to glutathione beads.

383 Only anti- $\alpha$-tubulin and anti-VCL antibodies were of mouse origin; thus, the result is clear-cut 384 and cannot have been affected by successive membrane stripping, demonstrating the existence of 385 a PARylated VCL pool. Given the AP was done in the absence of crosslinking and in the 386 presence of high detergent and salt concentrations, non-covalent interactions were not expected 387 to be maintained. This was reflected in the fact that other proteins of the adherens junctions 388 complex were not affinity precipitated. Thus, this result indicated the existence of covalently 389 PARylated VCL. 
390 VCL was also affinity precipitated as a light band in experiments carried using the Af1521

391 Macrodomain resin, which recognizes mono or poly(ADP-ribosylated) proteins. PARP-1 was

392 also checked as an AP control. While the specific TNKSi G007LK hindered only VCL affinity

393 precipitation (Fig. S 2 A), XAV939, which inhibits PARP-1/2/3 \& TNKS, hampered both VCL

394 and PARP-1 affinity precipitation (Fig. S 2 B).

395 Altogether, these results support that TNKS is responsible for VCL PARylation.

396 To map the subcellular localization of PARylated VCL, we evaluated the co-occurrence of PAR 397 and VCL signals in confocal ICF images. Fig. 6 B is a scheme representing the relative position 398 of the subsequent confocal epithelial monolayer sections, running from basal to apical direction shown as a merged channels montage in Fig. $6 \mathrm{C}-\mathbf{H}$. The correspondent masks and multiplyied masks montages (Fig. 6 I - N and Fig. $6 \mathbf{O}$ - T) are also displayed to highlight the overlapping points. VCL was present in all these sections but the PAR belt was distributed along a 1 to 1.5 $\mu \mathrm{m}$ in $\mathrm{z}$ ( 3 to 5 slices depending on voxel height). PAR and VCL signals overlap drew just a thin polygon (in this case, a pentagon) representing the epithelial belt. No PARylation was ever detected at VCL-rich focal contacts (neither in untreated or treated cell cultures of Vero, NMuMG or MCF-7 cells, nor in mice epithelial tissues)

To quantify these observations, colocalization was analyzed in terms of signals overlap or signal 407 intensity correlation (see Materials and Methods).

408

409

410

411

412

413

414

415

416

417

418

We have included an example of intensity correlation analysis, taking a ROI from Fig. 6 E. The ROI red and green channel images (Fig. $6 \mathbf{U}$ and $\mathbf{V}$ ) were subjected to an intensity correlation analysis. Pearson Coefficient (Rr), which ranges from -1 to +1 (from anti-colocalization to complete colocalization), was 0.191. Li's ICQ (which ranges from -0.5 to +0.5 ) was 0.235 .

Double positive PDMs (products of the differences from the means) were localized precisely in the belt region (Fig. $6 \mathbf{W}$ ), just as the mask obtained through the correspondent masks multiplication (Fig. $6 \mathbf{X}$ ). Therefore, the intensity correlation analysis and the overlapping analysis converged in the epithelial belt region. Thus, mask multiplication to highlight overlapping signals and the overlapping analysis was used hereon (see supplementary Fig. S3 AL). We have additionally explored PAR and VCL colocalization in an EMT model. Epithelial NMuMG cells display PAR and VCL colocalization (overlap) just at the epithelial belt region 
419 (Fig. S3 M) which is lost upon treatment with TGF- $\beta$ to induce EMT (Fig. S3 N). ( For further 420 reference, see our previous work [30]). We have also analyzed PAR-VCL in belt ROIs 421 colocalization (overlap) focused on epithelial belt ROIs (Fig. 6 Y). In this case, Mander's 422 coefficient M1 ranged from 0.262 to 0.660 , indicating that $\approx 30$ to $60 \%$ of belt VCL signal 423 overlapped with PAR signal; M2 ranged from 0.255 to 0.882 indicating that $\approx 30$ to $90 \%$ of belt 424 PAR signal overlapped with VCL.

425 Partial colocalization of PAR and VCL (within $200 \mathrm{~nm}$ ) had also been previously observed in 426 Vero cells by ICF and confocal microscopy [15]. FRET was carried after indirect ICF (Fig. S4), 427 lowering the colocalization maximal distance to about $50 \mathrm{~nm}$ (estimated according König et al 4282006 [63]) as the addition of $10 \mathrm{~nm}$ FRET $+10 \mathrm{~nm}$ each primary antibody $+10 \mathrm{~nm}$ each 429 secondary antibody). FRET results were not included in the main manuscript because this 430 resolution did still not allow distinguishing among VCL and nearby PARylated proteins nor 431 among covalently PARylated or PAR-bound proteins; thus, the affinity precipitation result 432 constituted by far stronger evidence.

433 Not only not all VCL was PARylated (according to AP and ICF results), but also not all belt 434 PARylation could be attributed to VCL; the band pattern detected after subcellular fractionation 435 using the PAR-detecting reagent indicated there may be additional targets.

5. Mesenchymal-like shaped cells were observed under VCL/*TBM transient overexpression We finally wanted to study whether VCL PARylation was related to its functions. Using the obtained information and given the importance of the single conserved c-TBM in Drosophila to preserve its function, we decided to mutate the equivalent motif (c-TBM-II) in vertebrate VCL.

Direct mutagenesis of VCL in pET15b/GgVCL was achieved using primer pair G454V and the circular mutagenesis protocol suggested by Wang and Wilkinson (2000) [59] in the presence of DMSO. As depicted in Fig. 7 A, a ggc codon (coding Glycine) was substituted by gTA (coding Valine), generating an AccI (BioLabs R0161S) diagnostic restriction recognition site (gTAgac). Then, after incorporating each VCL sequence to a middle entry plasmid (pME), pME-VCL and pME-VCL/*TBM were obtained. The recombination of a plasmid carrying a $\beta$-actin promoter, pME-VCL or pME-VCL/*TBM, a plasmid coding an IRES-GFP and a destination plasmid

448 allowed to obtain the desired eukaryotic expression plasmids depicted in Fig. 7 B. 
449 Transfection of MCF-7 cells with Tol2-GgVCL (Fig. 8 A, B, E-J) or Tol2-GgVCL/*TBM (Fig. 4508 C, D, K - O) was achieved using FUGENE reagent on cell monolayers. These Tol2 plasmids 451 allow the identification of transfected cells that express the plasmid as GFP+ cells (Fig. 8 A'- O', 452 in green). Although carried in parallel and in the same conditions, the transfection with Tol2$453 \mathrm{GgVCL} / * \mathrm{TBM}$ rendered more GFP+ cells than the WT version, indicating that this transfection 454 was more effective or less cytotoxic (low cell numbers did not allow carrying additional 455 quantifications to distinguish among these two scenarios). Moreover, image intensity 456 quantifications showed that GFP expression was similar in Tol2-GgVCL or Tol2-GgVCL/*TBM 457 cells while VCL expression was doubled in the mutant with respect to the WT transfected cells. 458 It is worth noting that cells transfected with Tol2-GgVCL/*TBM presented a distorted 459 morphology without lineal tense cell junctions and with filopodia or lamellipodia, that looked 460 mesenchymal-like (Fig. 8 C, D, K - O). Cell shape was measured and the results are in Fig. 9. 461 While GgVCL transfected cells displayed a median CShin $<1$, cells transfected with $462 \mathrm{GgVCL} / * \mathrm{TBM}$ had a significatively higher CShin. This result strongly suggested the 463 involvement of TBM in the function of VCL to maintain cell shape.

464 To confirm this result, we aimed to use a KO-add back approach. We attempted to obtain VCL 465 knocked-out MCF cells by a CRISPR/Cas9 strategy (Fig. S5 A). Only a limited heterogeneous 466 cell pool could be obtained as transfected cells grew extremely slowly and lost their colony 467 forming ability under isolation (Fig. S5 B - Q, Fig. S6 A - H), precluding the obtainment of pure 468 clones and the possibility of straight-forward VCL KO confirmation. The available cells, 469 however, were transfected with the Tol2-GgVCL/*TBM plasmids (Fig. S7 A - N) or Tol2470 GgVCL (Fig. S7 O - R). CShin was sensitive enough to detect changes in cell shape induced by 471 the presumptive VCL partial "KO" which were reverted by the transfection with Gg WT VCL 472 but not with Gg VCL/*TBM (see contours examples in Fig. S7 E, I, L \& P, and the graph in 473 Fig. S8). Unfortunately, the obstacles regarding the "KO" cells obtainment limited the 474 conclusions that could be driven from these experiments and pointed out the need to implement 475 an alternative approach to confirm these observations. 


\section{Discussion}

477 Our hypothesis was that TNKS PARylates epithelial belt VCL, affecting adherens junctions

478 VCL-dependent functions, like subcortical actin ring anchorage and epithelial cell shape

479 maintenance. The insight to consider this pool of VCL as a putative PARylation target came

480 from ICF images and data obtained in Vero cells, where partial colocalization of belt PAR and

481 VCL was observed [15], indicating a distance $<200 \mathrm{~nm}$ among them. FRET allowed to shorten

482 the spatial proximity radio from PAR to VCL (or from VCL to PAR) to $<50 \mathrm{~nm}$ (Fig. S4). The

483 hypothesis that TNKS could be the PARylating enzyme was based on previous works indicating

484 the role of TNKS in cell junctions [40,41]. We performed a sequence analysis that revealed that

485 human VCL harbored three TBMs: two c-TBM and one nc-TBM. In 2011 Guetler and

486 collaborators reported a huge list of TMB bearing proteins. VCL was included, but at that time

487 only the canonical TBMs were reported [42]. TBMs presence is a requirement to be a TNKS

488 target. For this reason, the presence of TBMs reinforced the hypothesis regarding both VCL as a

489 PARylation target and TNKS as the involved enzyme. Moreover, the three detected TBMs were

490 conserved among well-studied vertebrate models including green monkey, mice, chicken and

491 zebrafish. Among invertebrates, one of the TBMs was present in VCL from Drosophila, whose

492 adherens junctions are quite similar to those of vertebrates, while none of the TBMs was found

493 in Caenorhabditis elegans. Interestingly, "in contrast to vertebrates, C. elegans adherens

494 junction proteins are not essential for general cell adhesion or for epithelial cell polarization"

495 [64]. Thus, a comparative analysis suggested that, although the presence of more than one TBM

496 may facilitate the establishment of stronger interactions with TNKS, at least one of the VCL

497 TBMs was deeply involved in adherens junctions functions and was highly conserved. A simple

498 sequence alignment of human/vertebrate vs Drosophila VCL yielded two possible results,

499 pointing to TBM-I or TBM-II (Fig. 2 A). In silico modeling of Drosophila VCL allowed us to

500 observe that TBM-II in human VCL is a loop localized like Drosophila single TBM (Fig. 2 B).

501 The binding of a substrate protein by ARCs is required for subsequent PARylation, but may not

502 be sufficient for the modification, as certain substrates are not modified upon binding.

503 PAR association to the plasma membrane was confirmed through a biochemical approach, using

504 the PAR-binding reagent MABE1031 to detect PAR in a plasma membrane fraction (PMF) of

505 Vero cells (Fig. 3 A). These results agree with the recent detection of enriched cortical PAR in 
506 mouse oocyte meiosis [65] and confirm the ones previously obtained combining ICF with either 507 post-fixation PAR digestion with PARG or pretreatments of cells with XAV939 in Vero cells 508 [15]. XAV939 was formerly thought to be a specific TNKS inhibitor [66-68]. Therefore, data 509 relying on XAV939 were interpreted as favoring a role of TNKS-1/2 in PAR belt synthesis [15] 510 that fitted with the results obtained overexpressing TNKS in MDCK cells [40]. Nevertheless, it 511 has been recently demonstrated that XAV939 does also inhibit full length PARP-1 and PARP-2

512 [69], raising again the question of which is the PARP responsible for the synthesis of the PAR 513 belt. Experiments with more specific TNKS-1/2 inhibitors supported TNKS involvement in PAR 514 belt synthesis (Fig. 4). Additionally, TNKS was detected in the plasma membrane fraction of 515 Vero cells (Fig. 3 A - D).

516 Finally, affinity precipitation of covalently PARylated proteins was done using WWE affinity 517 resin (Fig. 6 A) or Macrodomain resin in the absence and presence of TNKSi (Fig. S2 A, B).

518 Clear-cut results were obtained demonstrating that a VCL fraction was precipitated while cortical 519 actin or other components of adherens junctions, like E-cadherin or $\beta$-catenin, were not. Both the 520 experimental conditions (precipitation under extremely astringent conditions and in the absence 521 of cross-linking) and the fact that VCL partners from adherens junction complexes were not 522 affinity precipitated argue in favor of a covalent VCL PARylation. This, together with the fact 523 that VCL harbors TBMs and that cell shape changes if a single aminoacid in a single TBM is 524 substituted, precluding a putative PARylation by TNKS, demonstrates that VCL is a direct 525 TNKS target. VCL had formerly been detected in PAR-bound protein pools. Gagné et al [70,71] 526 detected hVCL (but not E-cadherin or $\beta$-catenin) in fractions immunoprecipitated with anti-PAR 527 antibodies followed by mass spectrometry [70,71]. Moreover, VCL was recently identified in a 528 pool of octomeric PAR ( 8-mer PAR) binding proteins but not among $\sim 40$-mer PAR binders, 529 using photoaffinity probes of defined lengths [72]. As TNKS synthetizes short chain PAR, it is 530 not surprising that VCL has been recovered among $~ 8$-mer PAR binders.

531 ICF confocal microscopy images allowed to compare VCL in focal adhesions vs VCL in the 532 adhesion belt, to observe that partial colocalization existed among belt VCL and PAR but there 533 was no PAR signal detected around the VCL located at focal adhesions (Fig. 6 C - Y, Fig. S3, 534 Fig. S4). Thus, the VCL pool PARylated by TNKS (Fig. 6 A \& Fig. S2) seemed to correspond 535 to the VCL pool bound to the adhesion belt (Fig. 6 C - Y, Fig S3, Fig. S4). 
536 Then, we further investigated the role of TBM in VCL on epithelial cells.

537 To be able to compare cell shape changes, a Cell Shape index was designed and validated using

538 an EMT model. CShin proved to be a robust cell shape quantification method and coherent 539 results were obtained. The comparison of graphs in Fig. 1 (NMuMG and EMT), Fig.5 (TNKSi

540 effects), Fig. 9 and Fig. S8 ("KO"and GgVCL vs GgVCL/*TBM effects) indicates that in all

541 cases epithelial cells displayed a median CShin $<1$, while CShin increased similarly under

542 treatment by TNKSi, VCL "KO" or VCL/*TBM indicated that any interference on TNKS and

543 VCL interaction has effects on cell shape similar to those observed in the EMT model.

544 VCL directed mutagenesis and overexpression of Tol2-GgVCL or Tol2-VCL/*TBM in MCF-7

545 human epithelial breast cancer cells showed that $\mathrm{VCL} / * \mathrm{TBM}$ induced mesenchymal-like changes

546 in cell morphology that were more similar to those expected under knockdown conditions than

547 under VCL overexpression, suggesting that the TBM could be crucial to VCL function in cell

548 junctions (Fig. 8, Fig. 9, Fig. S8).

549 Our transfections with Tol2-GgVCL or Tol2-GgVLC/*TBM had important design limitations

550 that could be solved using a different approach. First, it was meant to achieve transient

551 exogenous GgVCL expression, making it impossible to follow up cells in migration, invasion or

552 chemo-resistance studies. Second, even though we chose a medium-expression promoter ( $\beta$-actin

553 promoter rather than the stronger CMV), there was a clear GgVCL overexpression. It is worth

554 trying a direct substitution of nucleotides on the endogenous VCL (to obtain G454V aminoacidic

555 change), so that the endogenous VCL gene expression remains unchanged. A long-term

556 expression of TBM*/hVLC, without a change in basal expression level could be achieved

557 through CRISPR-directed edition of the endogenous hVCL gene in MCF-7 cells. In spite of the

558 fact that VCL harbors at least 3 TBMs, we have observed promising interesting changes in cell

559 shape introducing a single aminoacidic substitution in TBM-II.

\section{Conclusions}

561 In this work, we started to test the hypothesis that TNKS PARylates epithelial belt VCL,

562 affecting adherens junctions VCL-dependent functions. To sum up, the hypothesis has several

563 predictions which have been corroborated: (1) VCL harbored TBMs conserved in evolution 
564 correlating with adherens junctions functions; (2) TNKS inhibitors disrupted the PAR belt while

565 PAR and a TNKS pool was detected in the plasma membrane fraction: (3) A VCL pool was

566 covalently PARylated in the absence of TNKSi (4) overexpression of VCL/*TBM induced

567 changes in cell morphology remnant of an EMT, which have been described in the context of

568 VCL knockdown [16] or VCL loss [73].

569 The last important prediction of our hypothesis would be that (5) the effect of long-term

570 expression of VCL/*TBM at physiological levels on cell junctions would be EMT induction,

571 implying changes on epithelial morphology, migration and invasion capacity as well as chemo-

572 resistance. This last prediction remains to be tested. Importantly, as the adherens junctions have

573 distinct distribution and roles in certain non-epithelial tissues [62,74], any discovery regarding

574 adherens junctions composition and functions is putatively exciting in fields that look as distant

575 as cancer, infections, neurodegeneration or spermatogenesis. Several processes in different cell

576 types could be modulated by TBM-dependent VCL PARylation by TNKS. Thus, we hope this

577 work will encourage further research in diverse areas.

\section{Acknowledgements}

579 We are grateful to everyone who made this work possible. MCF7 cells were a generous gift from 580 Dr. Archana Dhasarathy and Dr. Sergei Nechaev, University of North Dakota, USA, and were 581 routinely cultured at the Cell Culture Room from the Neurochemistry Department, IIBCE. Vero

582 cells were donated by Dr. Santiago Mirazzo and Dr. Juan Arbiza. AccI was a kind gift from Dr.

583 Fernanda Azpiroz, from Microbiology Laboratory at Science Faculty, UdelaR. Anti-PMCA and 584 anti-GRASP antibodies were respectively donated by Dr. Gerardo Corradi, IQUIFIB-CONICET 585 and Dr. Cynthia He Yingxin, National University of Singapore. Dr. Lari Lehtio gently gave us 586 TNKSi. Dr. Marcelo Comini and Dr. Mariana Bonilla gave us open access to the Lonza

587 Nucleofector and the cell culture room at IPMONT. We are particularly indebted to Dr José 588 Badano and his team, including Dr Victoria Prieto and Dr Paola Lepanto, who opened their 589 laboratory at IPMONT and shared with us their reagents, their molecular biology background 590 and their valuable time. We are also grateful to Synthego who donated us the CRISPR/Cas9 591 VCL KO kit. 


\section{References}

593 1. Balda, M.S.; Matter, K. Epithelial Cell Adhesion and the Regulation of Gene Expression.

594

595

596

597

598

599

600

601

602

603

604

605

606

607

608

609

610

611

612

613

614

615

616

617

618

619

620

621

622

623

624

625

626

627

628

629

630

631

632

633

634

635

636

637

638

639

2003, 13, 310-318, doi:10.1016/S0962-8924(03)00105-3.

2. Aho, S.; Lupo, J.; Coly, P.; Castellazzi, M.; Morand, P.; Gruffat, H. Characterization of the Ubinuclein Protein as a New Member of the Nuclear and Adhesion Complex Components ( NACos ). 2009, 101, 319-334, doi:10.1042/BC20080072.

3. AJ Unknown Components_Niessen Gottardi 2008.Pdf.

4. Franke, W.W. Discovering the Molecular Components of Intercellular Junctions--a Historical View. Cold Spring Harbor perspectives in biology 2009, 1, a003061, doi:10.1101/cshperspect.a003061.

5. Carisey, A.; Ballestrem, C. European Journal of Cell Biology Vinculin , an Adapter Protein in Control of Cell Adhesion Signalling. European Journal of Cell Biology 2011, 90, 157163, doi:10.1016/j.ejcb.2010.06.007.

6. Su, Y.-J.; Chang, Y.-W.; Lin, W.-H.; Liang, C.-L.; Lee, J.-L. An Aberrant Nuclear Localization of E-Cadherin Is a Potent Inhibitor of Wnt/ß-Catenin-Elicited Promotion of the Cancer Stem Cell Phenotype. Oncogenesis 2015, 4, e157, doi:10.1038/oncsis.2015.17.

7. Kalluri, R.; Weinberg, R. a Review Series The Basics of Epithelial-Mesenchymal Transition. Journal of Clinical Investigation 2009, 119, 1420-1428, doi:10.1172/JCI39104.1420.

8. Dongre, A.; Weinberg, R.A. New Insights into the Mechanisms of Epithelial-Mesenchymal Transition and Implications for Cancer. Nature Reviews Molecular Cell Biology 2018, doi:10.1038/s41580-018-0080-4.

9. Tsubakihara, Y.; Moustakas, A. Epithelial-Mesenchymal Transition and Metastasis under the Control of Transforming Growth Factor $\beta$. International Journal of Molecular Sciences 2018, 19, 3672, doi:10.3390/ijms19113672.

10. VIRAG, L.; SZABO, C. The Therapeutic Potential of Poly(ADP-Ribose) Polymerase Inhibitors. Journal of the National Cancer Institute 2011, 103, 334-46, doi:10.1093/jnci/djq509.

11. Daniels, C.M.; Ong, S.E.; Leung, A.K.L. The Promise of Proteomics for the Study of ADPRibosylation. Molecular Cell 2015, 58, 911-924, doi:10.1016/j.molcel.2015.06.012.

12. Barkauskaite, E.; Jankevicius, G.; Ahel, I. Structures and Mechanisms of Enzymes Employed in the Synthesis and Degradation of PARP-Dependent Protein ADP-

Ribosylation. Molecular Cell 2015, 58, 935-946, doi:10.1016/j.molcel.2015.05.007.

13. Hottiger, M.O. SnapShot: ADP-Ribosylation Signaling. Molecular cell 2015, 58, 11341134, doi:10.1016/j.molcel.2015.06.001.

14. Leung, A.K.L. Poly(ADP-Ribose): An Organizer of Cellular Architecture. The Journal of Cell Biology 2014, 205, 613-619, doi:10.1083/jcb.201402114.

15. Lafon-Hughes, L.; Vilchez Larrea, S.C.; Kun, A.; Fernández Villamil, S.H. VERO Cells Harbor a Poly-ADP-Ribose Belt Partnering Their Epithelial Adhesion Belt. PeerJ 2014, 2, e617, doi:10.7717/peerj.617.

16. Rodríguez Fernández, J.; Geiger, B.; Salomon, D.; Ben-Ze'ev, A. Suppression of Vinculin Expression by Antisense Transfection Confers Changes in Cell Morphology, Motility, and Anchorage-Dependent Growth of 3T3 Cells. The Journal of Cell Biology 1993, 122, 12851294, doi:10.1083/jcb.122.6.1285.

17. Pal, I.; Rajesh, Y.; Banik, P.; Dey, G.; Dey, K.K.; Bharti, R.; Naskar, D.; Chakraborty, S.; Ghosh, S.K.; Das, S.K.; et al. Prevention of Epithelial to Mesenchymal Transition in Colorectal Carcinoma by Regulation of the E-Cadherin- $\beta$-Catenin-Vinculin Axis. Cancer Letters 2019, 452, 254-263, doi:10.1016/j.canlet.2019.03.008. 
640

641

642

643

644

645

646

647

648

649

650

651

652

653

654

655

656

657

658

659

660

661

662

663

664

665

666

667

668

669

670

671

672

673

674

675

676

677

678

679

680

681

682

683

684

685

686

687

688

689

18. Peng, X.; Cuff, L.E.; Lawton, C.D.; DeMali, K.A. Vinculin Regulates Cell-Surface ECadherin Expression by Binding to -Catenin. Journal of Cell Science 2010, 123, 567-577, doi:10.1242/jcs.056432.

19. Mierke, C.T.; Kollmannsberger, P.; Zitterbart, D.P.; Diez, G.; Koch, T.M.; Marg, S.; Ziegler, W.H.; Goldmann, W.H.; Fabry, B. Vinculin Facilitates Cell Invasion into Three-Dimensional. 2010, 285, 13121-13130, doi:10.1074/jbc.M109.087171.

20. Rahman, A.; Carey, S.P.; Kraning-rush, C.M.; Goldblatt, Z.E.; Lampi, M.C.; Lin, D.Y.; García, A.J.; Reinhart-king, C.A. Vinculin Regulates Directionality and Cell Polarity in Twoand Three-Dimensional Matrix and Three-Dimensional Microtrack Migration. 2016, 27 , doi:10.1091/mbc.E15-06-0432.

21. Bays, J.L.; Demali, K.A. Vinculin in Cell - Cell and Cell - Matrix Adhesions. 2017, 29993009, doi:10.1007/s00018-017-2511-3.

22. Rüdiger, M. Vinculin and Alpha-Catenin: Shared and Unique Functions in Adherens Junctions. Bioessays 1998, 20, 733-740, doi:10.1002/(SICl)15211878(199809)20:9<733::AID-BIES6>3.0.CO;2-H.

23. Raz, A.; Geiger, B. Altered Organization of Cell-Substrate Contacts and MembraneAssociated Cytoskeleton in Tumor Cell Variants Exhibiting Different Metastatic Capabilities. Cancer Res. 1982, 42, 5183-5190.

24. Coll, J.L.; Ben-Ze'ev, A.; Ezzell, R.M.; Rodriguez Fernandez, J.L.; Baribault, H.; Oshima, R.G.; Adamson, E.D. Targeted Disruption of Vinculin Genes in F9 and Embryonic Stem Cells Changes Cell Morphology, Adhesion, and Locomotion. Proceedings of the National Academy of Sciences 1995, 92, 9161-9165, doi:10.1073/pnas.92.20.9161.

25. Xu, W.; Coll, J.L.; Adamson, E.D. Rescue of the Mutant Phenotype by Reexpression of Full-Length Vinculin in Null F9 Cells; Effects on Cell Locomotion by Domain Deleted Vinculin. J. Cell. Sci. 1998, 111 ( Pt 11), 1535-1544.

26. Sumida, G.M.; Tomita, T.M.; Shih, W.; Yamada, S. Myosin II Activity Dependent and Independent Vinculin Recruitment to the Sites of E-Cadherin-Mediated Cell-Cell Adhesion. BMC Cell Biology 2011, 12, 48, doi:10.1186/1471-2121-12-48.

27. Maddugoda, M.P.; Crampton, M.S.; Shewan, A.M.; Yap, A.S. Myosin VI and Vinculin Cooperate during the Morphogenesis of Cadherin Cell-Cell Contacts in Mammalian Epithelial Cells. Journal of Cell Biology 2007, 178, 529-540, doi:10.1083/jcb.200612042.

28. Hwang, J.R.; Chou, C.-L.; Medvar, B.; Knepper, M.A.; Jung, H.J. Identification of $\beta$ Catenin-Interacting Proteins in Nuclear Fractions of Native Rat Collecting Duct Cells. American Journal of Physiology-Renal Physiology 2017, 313, F30-F46, doi:10.1152/ajprenal.00054.2017.

29. Flachs, P.; Darasova, A.; Hozak, P. Nuclear Vinculin Involvement in Mouse Spermatogenesis. Biopolym. Cell 2019, 35, 177-178, doi:10.7124/bc.0009B4.

30. Schacke, M.; Kumar, J.; Colwell, N.; Hermanson, K.; Folle, G.; Nechaev, S.; Dhasarathy, A.; Lafon-Hughes, L. PARP-1/2 Inhibitor Olaparib Prevents or Partially Reverts EMT Induced by TGF- $\beta$ in NMuMG Cells. International Journal of Molecular Sciences 2019, 20 , 518, doi:10.3390/ijms20030518.

31. Hottiger, M.O.; Hassa, P.O.; Lüscher, B.; Schüler, H.; Koch-Nolte, F. Toward a Unified Nomenclature for Mammalian ADP-Ribosyltransferases. Trends in Biochemical Sciences 2010, 35, 208-219, doi:10.1016/j.tibs.2009.12.003.

32. Vyas, S.; Matic, I.; Uchima, L.; Rood, J.; Zaja, R.; Hay, R.T. Europe PMC Funders Group Family-Wide Analysis of Poly ( ADP-Ribose ) Polymerase Activity. 2015, 1-29, doi:10.1038/ncomms5426.Family-wide.

33. Dantzer, F.; Santoro, R. The Expanding Role of PARPs in the Establishment and Maintenance of Heterochromatin. FEBS Journal 2013, 280, 3508-3518, doi:10.1111/febs.12368.

PeerJ reviewing PDF | (2020:10:54544:2:0:CHECK 16 Apr 2021) 
690

691

692

693

694

695

696

697

698

699

700

701

702

703

704

705

706

707

708

709

710

711

712

713

714

715

716

717

718

719

720

721

722

723

724

725

726

727

728

729

730

731

732

733

734

735

736

737

738

739

740

34. Lafon-Hughes, L.; Di Tomaso, M.V.; Méndez-Acuña, L.; Martínez-López, W. ChromatinRemodelling Mechanisms in Cancer. Mutation Research - Reviews in Mutation Research 2008, 658, 191-214, doi:10.1016/j.mrrev.2008.01.008.

35. Vyas, S.; Chesarone-Cataldo, M.; Todorova, T.; Huang, Y.-H.; Chang, P. A Systematic Analysis of the PARP Protein Family Identifies New Functions Critical for Cell Physiology. Nature Communications 2013, 4, doi:10.1038/ncomms3240.

36. Bottone, M.G.; Santin, G.; Soldani, C.; Veneroni, P.; Alpini, C.; Scovassi, A.I. Intracellular Distribution of Tankyrases as Detected by Multicolor Immunofluorescence Techniques. European Journal of Histochemistry 2012, 56, 20-23, doi:10.4081/ejh.2012.e4.

37. Chi, N.W.; Lodish, H.F. Tankyrase Is a Golgi-Associated Mitogen-Activated Protein Kinase Substrate That Interacts with IRAP in GLUT4 Vesicles. Journal of Biological Chemistry 2000, 275, 38437-38444, doi:10.1074/jbc.M007635200.

38. Hsiao, S.J.; Smith, S. Sister Telomeres Rendered Dysfunctional by Persistent Cohesion Are Fused by NHEJ. Journal of Cell Biology 2009, 184, 515-526, doi:10.1083/jcb.200810132.

39. Bhardwaj, A.; Yang, Y.; Ueberheide, B.; Smith, S. Whole Proteome Analysis of Human Tankyrase Knockout Cells Reveals Targets of Tankyrase-Mediated Degradation. Nat Commun 2017, 8, 2214, doi:10.1038/s41467-017-02363-w.

40. Yeh, T.-Y.J.; Meyer, T.N.; Schwesinger, C.; Tsun, Z.-Y.; Lee, R.M.; Chi, N.-W. Tankyrase Recruitment to the Lateral Membrane in Polarized Epithelial Cells: Regulation by Cell-Cell Contact and Protein Poly(ADP-Ribosyl)Ation. Biochemical Journal 2006, 399, 415-425, doi:10.1042/BJ20060713.

41. Campbell, C.I.; Samavarchi-Tehrani, P.; Barrios-Rodiles, M.; Datti, A.; Gingras, A.-C.; Wrana, J.L. The RNF146 and Tankyrase Pathway Maintains the Junctional Crumbs Complex through Regulation of Angiomotin. J Cell Sci 2016, 129, 3396-3411, doi:10.1242/jcs.188417.

42. Guettler, S.; Larose, J.; Petsalaki, E.; Gish, G.; Scotter, A.; Pawson, T.; Rottapel, R.; Sicheri, F. Structural Basis and Sequence Rules for Substrate Recognition by Tankyrase Explain the Basis for Cherubism Disease. Cell 2011, 147, 1340-1354, doi:10.1016/j.cell.2011.10.046.

43. DaRosa, P.A.; Klevit, R.E.; Xu, W. Structural Basis for Tankyrase-RNF146 Interaction Reveals Noncanonical Tankyrase-Binding Motifs: Structural Basis for Tankyrase-RNF146 Interaction. Protein Science 2018, 27, 1057-1067, doi:10.1002/pro.3413.

44. Pollock, K.; Ranes, M.; Collins, I.; Guettler, S. Identifying and Validating Tankyrase Binders and Substrates: A Candidate Approach. In Poly(ADP-Ribose) Polymerase; Tulin, A.V., Ed.; Methods in Molecular Biology; Springer New York: New York, NY, 2017; Vol. 1608, pp. 445-473 ISBN 978-1-4939-6992-0.

45. Eisemann, T.; Langelier, M.-F.; Pascal, J.M. Structural and Functional Analysis of Parameters Governing Tankyrase-1 Interaction with Telomeric Repeat-Binding Factor 1 and GDP-Mannose 4,6-Dehydratase. J. Biol. Chem. 2019, 294, 14574-14590, doi:10.1074/jbc.RA119.009200.

46. Haikarainen, T.; Koivunen, J.; Narwal, M.; Venkannagari, H.; Obaji, E.; Joensuu, P.; Pihlajaniemi, T.; Lehtiö, L. Para-Substituted 2-Phenyl-3,4-Dihydroquinazolin-4-Ones as Potent and Selective Tankyrase Inhibitors. ChemMedChem 2013, 8, 1978-1985, doi:10.1002/cmdc.201300337.

47. Haikarainen, T.; Krauss, S.; Lehtio, L. Tankyrases: Structure, Function and Therapeutic Implications in Cancer. Current Pharmaceutical Design 2014, 20, 6472-6488, doi:10.2174/1381612820666140630101525.

48. Riffell, J.L.; Lord, C.J.; Ashworth, A. Tankyrase-Targeted Therapeutics: Expanding Opportunities in the PARP Family. Nature Reviews Drug Discovery 2012, 11, 923-936, doi:10.1038/nrd3868.

Peer) reviewing PDF | (2020:10:54544:2:0:CHECK 16 Apr 2021) 
741

742

743

744

745

746

747

748

749

750

751

752

753

754

755

756

757

758

759

760

761

762

763

764

765

766

767

768

769

770

771

772

773

774

775

776

777

778

779

780

781

782

783

784

785

786

787

788

789

790

49. Lehtiö, L.; Chi, N.W.; Krauss, S. Tankyrases as Drug Targets. FEBS Journal 2013, 280, 3576-3593, doi:10.1111/febs.12320.

50. Mariotti, L.; Pollock, K.; Guettler, S. Regulation of Wnt/ß-Catenin Signalling by TankyraseDependent Poly(ADP-Ribosyl)Ation and Scaffolding. British Journal of Pharmacology 2017, 174, 4611-4636, doi:10.1111/bph.14038.

51. Thorsell, A.-G.; Ekblad, T.; Karlberg, T.; Löw, M.; Pinto, A.F.; Trésaugues, L.; Moche, M.; Cohen, M.S.; Schüler, H. Structural Basis for Potency and Promiscuity in Poly(ADPRibose) Polymerase (PARP) and Tankyrase Inhibitors. Journal of Medicinal Chemistry 2017, 60, 1262-1271, doi:10.1021/acs.jmedchem.6b00990.

52. Haikarainen, T.; Waaler, J.; Ignatev, A.; Nkizinkiko, Y.; Venkannagari, H.; Obaji, E.; Krauss, S.; Lehtiö, L. Development and Structural Analysis of Adenosine Site Binding Tankyrase Inhibitors. Bioorganic and Medicinal Chemistry Letters 2016, 26, 328-333, doi:10.1016/j.bmcl.2015.12.018.

53. Innocenti, M. New Insights into the Formation and the Function of Lamellipodia and Ruffles in Mesenchymal Cell Migration. Cell Adhesion \& Migration 2018, 1-16, doi:10.1080/19336918.2018.1448352.

54. Larkin, M.A.; Blackshields, G.; Brown, N.P.; Chenna, R.; McGettigan, P.A.; McWilliam, H.; Valentin, F.; Wallace, I.M.; Wilm, A.; Lopez, R.; et al. Clustal W and Clustal X Version 2.0. Bioinformatics 2007, 23, 2947-2948, doi:10.1093/bioinformatics/btm404.

55. Han, M.K.L.; van der Krogt, G.N.M.; de Rooij, J. Zygotic Vinculin Is Not Essential for Embryonic Development in Zebrafish. PLOS ONE 2017, 12, e0182278, doi:10.1371/journal.pone.0182278.

56. Huang, X.; Miller, W. A Time-Efficient, Linear-Space Local Similarity Algorithm. Advances in Applied Mathematics 1991, 12, 337-357, doi:10.1016/0196-8858(91)90017-D.

57. Nielsen, M.; Lundegaard, C.; Lund, O.; Petersen, T.N. CPHmodels-3.0-Remote Homology Modeling Using Structure-Guided Sequence Profiles. Nucleic Acids Research 2010, 38, W576-W581, doi:10.1093/nar/gkq535.

58. Krieger, E.; Koraimann, G.; Vriend, G. Increasing the Precision of Comparative Models with YASARA NOVA-a Self-Parameterizing Force Field. Proteins 2002, 47, 393-402, doi:10.1002/prot.10104.

59. Wang, J.; Wilkinson, M.F. Site-Directed Mutagenesis of Large (13-Kb) Plasmids in a Single-PCR Procedure. BioTechniques 2000, 29, 976-978, doi:10.2144/00295bm09.

60. Kwan, K.M.; Fujimoto, E.; Grabher, C.; Mangum, B.D.; Hardy, M.E.; Campbell, D.S.; Parant, J.M.; Yost, H.J.; Kanki, J.P.; Chien, C.-B. The Tol2kit: A Multisite Gateway-Based Construction Kit ForTol2 Transposon Transgenesis Constructs. Dev. Dyn. 2007, 236, 3088-3099, doi:10.1002/dvdy.21343.

61. Yagita, K.; Yamanaka, I.; Emoto, N.; Kawakami, K.; Shimada, S. Real-Time Monitoring of Circadian Clock Oscillations in Primary Cultures of Mammalian Cells Using Tol2 Transposon-Mediated Gene Transfer Strategy. BMC Biotechnol 2010, 10, 3, doi:10.1186/1472-6750-10-3.

62. Lafon Hughes, L.I.; Romeo Cardeillac, C.J.; Cal Castillo, K.B.; Vilchez Larrea, S.C.; Sotelo Sosa, J.R.; Folle Ungo, G.A.; Fernández Villamil, S.H.; Kun González, A.E. Poly(ADPRibosylation) Is Present in Murine Sciatic Nerve Fibers and Is Altered in a Charcot-MarieTooth-1E Neurodegenerative Model. PeerJ 2017, 5, e3318, doi:10.7717/peerj.3318.

63. Konig, P.; Krasteva, G.; Tag, C.; Konig, I.R.; Arens, C.; Kummer, W. FRET-CLSM and Double-Labeling Indirect Immunofluorescence to Detect Close Association of Proteins in Tissue Sections. Laboratory investigation; a journal of technical methods and pathology 2006, 86, 853-864, doi:10.1038/labinvest.3700443.

64. Armenti, S.T.; Nance, J. Adherens Junctions in C. elegans Embryonic Morphogenesis. In Adherens Junctions: from Molecular Mechanisms to Tissue Development and Disease; 
791

792

793

794

795

796

797

798

799

800

801

802

803

804

805

806

807

808

809

810

811

812

813

814

815

816

817

818

819

820

821

822

823

824

825

826

827

828

829

830

831

832 index.

Harris, T., Ed.; Subcellular Biochemistry; Springer Netherlands: Dordrecht, 2012; Vol. 60, pp. 279-299 ISBN 978-94-007-4185-0.

65. Xie, B.; Zhang, L.; Zhao, H.; Bai, Q.; Fan, Y.; Zhu, X.; Yu, Y.; Li, R.; Liang, X.; Sun, Q.-Y.; et al. Poly(ADP-Ribose) Mediates Asymmetric Division of Mouse Oocyte. Cell Res 2018, 28, 462-475, doi:10.1038/s41422-018-0009-7.

66. Riffell, J.L.; Lord, C.J.; Ashworth, A. Tankyrase-Targeted Therapeutics: Expanding Opportunities in the PARP Family. Nature Reviews Drug Discovery 2012, 11, 923-936, doi:10.1038/nrd3868.

67. Lehtiö, L.; Chi, N.W.; Krauss, S. Tankyrases as Drug Targets. FEBS Journal 2013, 280, 3576-3593, doi:10.1111/febs.12320.

68. Haikarainen, T.; Krauss, S.; Lehtio, L. Tankyrases: Structure, Function and Therapeutic Implications in Cancer. Current pharmaceutical design 2014, 20, 6472-88, doi:10.2174/1381612820666140630101525.

69. Thorsell, A.G.; Ekblad, T.; Karlberg, T.; Löw, M.; Pinto, A.F.; Trésaugues, L.; Moche, M.; Cohen, M.S.; Schüler, H. Structural Basis for Potency and Promiscuity in Poly(ADPRibose) Polymerase (PARP) and Tankyrase Inhibitors. Journal of Medicinal Chemistry 2017, 60, 1262-1271, doi:10.1021/acs.jmedchem.6b00990.

70. Gagné, J.P.; Isabelle, M.; Lo, K.S.; Bourassa, S.; Hendzel, M.J.; Dawson, V.L.; Dawson, T.M.; Poirier, G.G. Proteome-Wide Identification of Poly(ADP-Ribose) Binding Proteins and Poly(ADP-Ribose)-Associated Protein Complexes. Nucleic Acids Research 2008, 36, 6959-6976, doi:10.1093/nar/gkn771.

71. Gagné, J.P.; Pic, É.; Isabelle, M.; Krietsch, J.; Éthier, C.; Paquet, É.; Kelly, I.; Boutin, M.; Moon, K.M.; Foster, L.J.; et al. Quantitative Proteomics Profiling of the Poly(ADP-Ribose)Related Response to Genotoxic Stress. Nucleic Acids Research 2012, 40, 7788-7805, doi:10.1093/nar/gks486.

72. Dasovich, M.; Beckett, M.Q.; Bailey, S.; Ong, S.-E.; Greenberg, M.M.; Leung, A.K.L. PARprolink: A Photoaffinity Probe for Identifying Poly(ADP-Ribose)-Binding Proteins; Molecular Biology, 2020;

73. Li, T.; Guo, H.; Song, Y.; Zhao, X.; Shi, Y.; Lu, Y.; Hu, S.; Nie, Y.; Fan, D.; Wu, K. Loss of Vinculin and Membrane-Bound $\beta$-Catenin Promotes Metastasis and Predicts Poor Prognosis in Colorectal Cancer. 2014, 1-15.

74. Lui, W.; Mruk, D.D.; Lee, W.M.; Cheng, C.Y. Adherens Junction Dynamics in the Testis and Spermatogenesis. Journal of andrology 2003, 24, 1-14.

\section{Figure Legends}

Figure 1. Cell Shape Index: design and proof of concept.

(A) Roundness, circularity and their quotients were initially measured and calculated for geometric figures. Here are CShin values for some geometric figures and the correspondent figures with a wavy limit. (B) NMuMG cells are a well-known model to study a process through which an epithelial cell loses its epithelial characteristics and acquires mesenchymal characteristics. Such process is an epithelial to mesenchymal transition (EMT) and is induced by a treatment with TGF- $\beta$. We induced that process and measured CShin to validate the proposed 
834 Figure 2. VCL sequence harbors three TBMs conserved in vertebrates, one of which is 835 present in Drosophila.

836 (A) VCL TBMs, according to the hexamer rule for c-TBMs or the heptamer rule for nc-TBMs, 837 follow the patterns: RXXOXG or RXXXOXG, where $\mathrm{X}$ is any aminoacid and $\mathrm{O}$ is $\mathrm{G}, \mathrm{P}, \mathrm{A}$ or $\mathrm{C}$. 838 These two patterns were searched and found conserved in vertebrates (Human, Mouse, Chicken, 839 Xenopus and Zebrafish) as indicated under TBM-I, TBM-II and TBM-III. Only one of them 840 was present in Drosophila while none of them was present in Caenorhabditis elegans. The single 841 TBM present in Drosophila can align with human TBM-I or TBM-II depending on the matrix 842 used for the alignment. (B) Superimposition of hVCL (blue, PDB: 1TR2, B subunit) and dVCL 843 predicted structure (grey). TBMs from hVCL are highlighted using the same color code than in 844 panel A, while Drosophila TBM is colored in pink. Overlapped hVCL and Drosophila VCL 845 structures evidence the equivalence of Drosophila TBM and vertebrate TBM-II.

846 Figure 3. Endogenous TNKS and PAR distribution in epithelial cells.

847 (A) Confluent Vero cells were subjected to subcellular fractionation. The identity of the obtained 848 fractions was confirmed using fractionation validation markers: for nuclear fraction (NF) Lamin $849 \mathrm{~A} / \mathrm{C}$, for cytoplasmic fraction (CF) CALR3, for internal membrane fraction (IMF) GRASP and 850 for plasma membrane fraction (PMF) PMCA. TNKS was more abundant in IMF, but was also 851 detected in the other cell fractions, including the PMF. PAR (detected with anti-PAR reagent 852 MABE-1031) was more abundant in the nuclear fraction but also detectable in the other cell 853 fractions. Arrows on the right indicate discrete bands corresponding to unknown PARylated 854 proteins observed in the PMF. (B - E) ICF to detect TNKS cellular localization (green), 855 counterstained with F-actin (red) and DAPI (blue). A non-symmetric perinuclear region probably 856 correspondent to the Golgi system harbored most TNKS. However, (D, E) a closer examination 857 evidenced a wider TNKS distribution, including plasma membrane or submembrane regions, as 858 indicated by white arrows. Bar: $25 \mu \mathrm{m}$.

859 Figure 4. TNKS inhibitors hampered PAR belt synthesis and altered the subcortical actin 860 ring.

861 TNKSi were added to Vero cells at the moment of seeding and after $5 \mathrm{~h}$ cells were fixed, washed 862 in PBS and subjected to ICF to detect PAR (green), F-actin with rhodamine-phalloidin 863 counterstain (red) and nuclei with DAPI (blue). (A - E) PAR channel; (F - J) F-actin channel, (K 
864 - O) merged channels. (A, F, K) control, (B, G, L) 80 nM FLALL-9, (C, H, M) 60 nM MN-64,

865 (D, I, N) $250 \mathrm{nM} \mathrm{G007-LK,} \mathrm{(E,} \mathrm{J,} \mathrm{O)} 2.6 \mu \mathrm{M}$ OD35. Instead of the tight straight continuous 866 appearance of the belt (A, F, K), a serrated discontinuous distribution was observed (B - O). Bar: $86725 \mu \mathrm{m}$. For a quantitative expression of cell shape change, see Fig. 5.

868 Figure 5. TNKSi changed cell shape

869 Cells were exposed to the PARP-1/2-3 inhibitor Olaparib (OLA) or to TNKSi FLALL, MN64, 870 G007LK or OD35, at the moment of seeding. Five hours later they were fixed after a quick wash 871 and subjected to ICF (see images in Fig. 4). Cell shape index was calculated as: CShin=

$872 \frac{\text { Roundness }}{\text { Circularity }}$. To appreciate the overall behavior of CShin and its validation as an index, see Fig. 1.

873 Figure 6. A VCL pool was PARylated

874 (A) Vero cell proteins were affinity precipitated using PAR-recognizing WWE-resin to recover 875 covalently-PARylated proteins. The flowthrough (FT) and affinity precipitated fractions (AP) 876 were subjected to WB. Five cell junction and cytoskeletal proteins were assayed. Unlike $\beta$ 877 catenin, E-cadherin, actin and $\alpha$-tubulin, a VCL pool was affinity precipitated (the arrow points 878 to the lower apparent MW VCL band, around 90 to $100 \mathrm{Kda}$, which was the most abundant in 879 the AP fraction). As expected, the resin did also precipitate PARylated PARP-1 and PAR itself. 880 The species in which each antibody was raised is indicated under the correspondent label 881 (R:rabbit; M:mouse) Except for mouse anti- $\alpha$-tubulin and mouse anti-VCL, all primary 882 antibodies used (anti- $\beta$-catenin, anti-E-cadherin, anti-PARP-1, anti-PAR) were of rabbit origin or 883 directly labeled (anti-actin). (B) Diagram indicative of the relative position of confocal images of 884 the right panels.( $\mathbf{C}-\mathbf{H})$ : ICF anti-VCL (red) and anti-PAR (green), merged channels; (I - N) 885 correspondent VCL and PAR masks; $(\mathbf{O}-\mathbf{T})$ : in yellow, masks product highlighting the 886 overlapping VCL and PAR regions, drawing precisely the epithelial belt. Subsequent confocal 887 planes, from basal to apical, allowed the distinction of VCL in focal adhesions (eg. C, I, O) from 888 VCL associated to the PAR belt (eg. G M, S). Bar: $25 \mu \mathrm{m}$. (U, V): VCL and PAR region of 889 interest around the belt (cropped from E). (W): ++PDMs from an intensity correlation 890 colocalization analysis. The pixels with values above the mean of each channel, in both channels, 891 are depicted. (X): in yellow, the product of $\mathbf{U}$ and $\mathbf{V}$ masks (as in $(\mathbf{O}-\mathbf{T})$. Notice that the 892 complex intensity correlation analysis and masks images multiplication lead to the same result: 893 colocalizing PAR and VCL pixels are on the epithelial belt. (Y): enlarged belt masks ROIs 
894 extracted from ( $\mathbf{L}$ - N), facilitate the precise visualization of colocalizing pixels. Overlapping 895 percentages were calculated according to Mander's coefficients (small numbers in Y: 1000x 896 Mander's coefficients).

897 Figure 7. Transfection of MCF-7 (P10) monolayer with Tol2 14.5 KB eukaryotic expression 898 vectors harboring VCL or VCL/*TBM.

899 (A) Circular PCR was carried on the 8909 pb plasmid template pET15b/GgVCL using $900 \mathrm{G} 454 \mathrm{~V}$ mutagenic primers to generate $\mathrm{pET} 15 \mathrm{~b} / \mathrm{GgVCL} / * \mathrm{TBM}$ (B) Schematic representation of 901 the $14.5 \mathrm{~KB}$ eukaryotic expression plasmids Tol2/Gg-VCL and Tol2/Gg-VCL/*TBM, 902 expressing the correspondent WT or mutated VCL under a $\beta$-actin promoter, plus green 903 fluorescent protein whose expression relied on an Internal Ribosome Entry Site (IRES-GFP). In 904 this way, transfected cells were expected to harbor free GFP (being easily identifiable) while 905 expressing untagged VCL.

906

907 Figure 8. Transfection with Tol2-GgVCL or Tol2-GgVCL*/TBM.

908 MCF-7 (P10) monolayers were transfected with either Tol2-GgVCL or Tol2-GgVCL/*TBM. 909 Cells were fixed $17 \mathrm{~h}$ later. GFP (green) and VCL (red) were detected by ICF. The left half of 910 the figure (A, B, E - J) corresponds to Tol2-GgVCL(WT), the right half $(\mathbf{C}, \mathbf{D}, \mathbf{K}-\mathbf{O})$ pertains 911 to Tol2-GgVCL/*TBM. (A - O): mixed channels; $\left(\mathbf{A}^{\prime}\right.$ - $\left.\mathbf{O}^{\prime}\right)$ : only GFP; (A" - $\left.\mathbf{O}^{\prime \prime}\right)$ : only VCL. 912 Notice the abundant VCL expression (neighbor cells in A are hardly detected) and the irregular, 913 mesenchymal-like shape of cells harboring mutated VCL. Bar: $25 \mu \mathrm{m}$.

914 Figure 9. Cell shape was different in cells transfected with WT or TBM*-GgVCL.

915 Cell shape index after transfection with WT VCL was below 1, as in other epithelial cells, while 916 transfection with VCL/*TBM significantly increased the index value. 
Table $\mathbf{1}$ (on next page)

Table 1. Antibodies and counterstains for ICF and WB. 


\begin{tabular}{|c|c|c|c|}
\hline code & Primary antibody/reagent & ICF & WB \\
\hline BD551813 & rabbit anti-PAR & $\begin{array}{l}1: 1500- \\
1: 50\end{array}$ & -- \\
\hline ENZO & rabbit anti-PAR & $1: 100$ & $1: 400$ \\
\hline ENZO BML-SA216 & mouse anti-PAR (<50 units) & $1: 50$ & $1: 1000$ \\
\hline MABE 1031 & rabbit anti-PAR reagent & -- & $1: 4000$ \\
\hline sc-7150 & rabbit anti-PARP & -- & $1: 4000$ \\
\hline GTX117417 & rabbit anti-TNKS-1/2 (Nt) & $1: 200$ & $1: 2000$ \\
\hline Novex 730101 & mouse anti-hTNKS; 1106-1125 & $1: 50$ & -- \\
\hline ab 73412 & rabbit anti-VCL (2016) & $1: 500$ & $1: 1500$ \\
\hline ab18058 & mouse anti-VCL (2013) & $1: 50$ & $1: 1000$ \\
\hline ab32572 & rabbit anti- $\beta$-catenin & $1: 1000$ & $1: 10000$ \\
\hline ab133597 & rabbit anti-E cadherin & -- & $1: 400$ \\
\hline STJ 93885 & rabbit anti-lamin $\mathrm{A} / \mathrm{C}$ & -- & $1: 4000$ \\
\hline Dr. Gerardo Corradi, IQUIFIB-CONICET & mouse anti-PMCA & -- & $1: 5000$ \\
\hline STJ190646 & rabbit anti-CALR & -- & $1: 2000$ \\
\hline $\begin{array}{l}\text { Dr. Cynthia He Yingxin, National } \\
\text { University of Singapore }\end{array}$ & anti-GRASP & -- & $1: 1000$ \\
\hline SIGMA & mouse anti- $\alpha$-tubulin & -- & $1: 10000$ \\
\hline sc-47778 HRP & mouse anti-actin -HRP & -- & $1: 10000$ \\
\hline Invitrogen A11122 & rabbit anti-GFP & $1: 1000$ & -- \\
\hline \multicolumn{4}{|c|}{ Secondary antibody or counterstain } \\
\hline ThermoFisher Sci goat anti-mouse 633 & goat anti-mouse 633 & $1: 500$ & -- \\
\hline Invitrogen A11034 & $\begin{array}{l}\text { goat anti-rabbit Alexa } 488 \\
\text { (A11034) }\end{array}$ & $1: 1000$ & -- \\
\hline Invitrogen A11030 & $\begin{array}{l}\text { goat anti-mouse Alexa Fluor } 546 \\
\text { (A11030); }\end{array}$ & $1: 1000$ & -- \\
\hline Invitrogen A11029 & goat anti-mouse Alexa 488 & $1: 1000$ & -- \\
\hline Invitrogen A11035 & goat anti-rabbit Alexa 546 & $1: 1000$ & -- \\
\hline Perkin Elmer & anti-rabbit-HRP & -- & $1: 6000$ \\
\hline Perkin Elmer & anti-mouse-HRP & -- & $1: 6000$ \\
\hline ab176756 & Cytopainter (actin cytoskeleton) & $1: 1000$ & -- \\
\hline Sigma-Aldrich & 4, 6-diamino-2-phenylindol (DAPI) & $\begin{array}{l}1, .5 \mu \mathrm{g} \\
/ \mathrm{mL}\end{array}$ & -- \\
\hline
\end{tabular}

1

2 Table 1. Antibodies and counterstains for ICF and WB.

3 


\section{Table 2 (on next page)}

Table 2. Potency of TNKS inhibitors towards PARP-1/2, TNKS-1/2 and TCPARP.

In vitro IC50 values determined for activity assays using full length (FL) or truncated recombinant enzymes. [1-7]

1. Haikarainen, T.; Koivunen, J.; Narwal, M.; Venkannagari, H.; Obaji, E.; Joensuu, P.; Pihlajaniemi, T.; Lehtiö, L. Para-substituted 2-phenyl-3,4-dihydroquinazolin-4-ones as potent and selective tankyrase inhibitors. ChemMedChem 2013, 8, 1978-1985, doi:10.1002/cmdc.201300337.

2. Haikarainen, T.; Krauss, S.; Lehtio, L. Tankyrases: Structure, Function and Therapeutic Implications in Cancer. Current Pharmaceutical Design 2014, 20, 6472-6488, doi:10.2174/1381612820666140630101525.

3. Riffell, J.L.; Lord, C.J.; Ashworth, A. Tankyrase-targeted therapeutics: expanding opportunities in the PARP family. Nature Reviews Drug Discovery 2012, 11, 923-936, doi:10.1038/nrd3868.

4. Lehtiö, L.; Chi, N.W.; Krauss, S. Tankyrases as drug targets. FEBS Journal 2013, 280, 3576-3593, doi:10.1111/febs.12320.

5. Mariotti, L.; Pollock, K.; Guettler, S. Regulation of Wnt/ß-catenin signalling by tankyrase-dependent poly(ADP-ribosyl)ation and scaffolding. British Journal of Pharmacology 2017, 174, 4611-4636, doi:10.1111/bph.14038.

6. Thorsell, A.-G.; Ekblad, T.; Karlberg, T.; Löw, M.; Pinto, A.F.; Trésaugues, L.; Moche, M.; Cohen, M.S.; Schüler, H. Structural Basis for Potency and Promiscuity in Poly(ADP-ribose) Polymerase (PARP) and Tankyrase Inhibitors. Journal of Medicinal Chemistry 2017, 60, 1262-1271, doi:10.1021/acs.jmedchem.6b00990.

7. Haikarainen, T.; Waaler, J.; Ignatev, A.; Nkizinkiko, Y.; Venkannagari, H.; Obaji, E.; Krauss, S.; Lehtiö, L. Development and structural analysis of adenosine site binding tankyrase inhibitors. Bioorganic and Medicinal Chemistry Letters 2016, 26, 328-333, doi:10.1016/j.bmcl.2015.12.018. 


\begin{tabular}{|c|c|c|c|c|}
\hline \multirow{2}{*}{$\begin{array}{c}\text { PARP } \\
\text { inhibitor }\end{array}$} & \multirow{2}{*}{ PARP-1 } & \multirow[b]{2}{*}{ PARP-2 } & \multirow[b]{2}{*}{ TNKS-1 } & \multirow[b]{2}{*}{ TNKS-2 } \\
\hline & & & & \\
\hline $\begin{array}{c}\text { FLALL } 9 \\
\text { (compound 8) }\end{array}$ & $\begin{array}{c}>10000 \\
{[46]}\end{array}$ & $\begin{array}{c}>10000 \\
{[46]}\end{array}$ & $5[46]$ & $\begin{array}{c}>10000 \\
{[46]}\end{array}$ \\
\hline MN64 & 19000 [47] & $35000[47]$ & $6[47]$ & 72 [47] \\
\hline XAV 939 & $\begin{array}{c}2200 \\
{[48,49]} \\
5500[50] \\
74(\mathrm{FL}) \\
{[51]}\end{array}$ & 27 [51] (FL) & $\begin{array}{c}11-13 \\
{[48,49]} \\
5[50] \\
95[51]\end{array}$ & $\begin{array}{c}4-5 \\
{[48,49]} \\
2[50] \\
5[51]\end{array}$ \\
\hline G007LK & - & - & $46[46]$ & 25 [46] \\
\hline $\begin{array}{c}\text { OD35 } \\
\text { (compound 2) }\end{array}$ & - & - & $11000[52]$ & 260 [52] \\
\hline
\end{tabular}

Table 2. Potency of TNKS inhibitors towards PARP-1/2 and TNKS-1/2. In vitro IC50 values determined for activity assays using full length $(F L)$ or truncated recombinant enzymes. All concentrations are expressed as nanomolar (nM).

46. Haikarainen, T.; Koivunen, J.; Narwal, M.; Venkannagari, H.; Obaji, E.; Joensuu, P.; Pihlajaniemi, T.; Lehtiö, L. Para-Substituted 2-Phenyl-3,4-Dihydroquinazolin-4-Ones as Potent and Selective Tankyrase Inhibitors. ChemMedChem 2013, 8, 1978-1985, doi:10.1002/cmdc.201300337.

47. Haikarainen, T.; Krauss, S.; Lehtio, L. Tankyrases: Structure, Function and Therapeutic Implications in Cancer. Current Pharmaceutical Design 2014, 20, 64726488, doi:10.2174/1381612820666140630101525.

48. Riffell, J.L.; Lord, C.J.; Ashworth, A. Tankyrase-Targeted Therapeutics:

Expanding Opportunities in the PARP Family. Nature Reviews Drug Discovery 2012, 11, 923-936, doi:10.1038/nrd3868.

49. Lehtiö, L.; Chi, N.W.; Krauss, S. Tankyrases as Drug Targets. FEBS Journal 2013, 280, 3576-3593, doi:10.1111/febs.12320.

50. Mariotti, L.; Pollock, K.; Guettler, S. Regulation of Wnt/ß-Catenin Signalling by Tankyrase-Dependent Poly(ADP-Ribosyl)Ation and Scaffolding. British Journal of Pharmacology 2017, 174, 4611-4636, doi:10.1111/bph.14038.

51. Thorsell, A.-G.; Ekblad, T.; Karlberg, T.; Löw, M.; Pinto, A.F.; Trésaugues, L.; Moche, M.; Cohen, M.S.; Schüler, H. Structural Basis for Potency and Promiscuity in Poly(ADP-Ribose) Polymerase (PARP) and Tankyrase Inhibitors. Journal of Medicinal Chemistry 2017, 60, 1262-1271, doi:10.1021/acs.jmedchem.6b00990.

52. Haikarainen, T.; Waaler, J.; Ignatev, A.; Nkizinkiko, Y.; Venkannagari, H.; Obaji, E.; Krauss, S.; Lehtiö, L. Development and Structural Analysis of Adenosine Site Binding Tankyrase Inhibitors. Bioorganic and Medicinal Chemistry Letters 2016, 26, 328-333, doi:10.1016/j.bmcl.2015.12.018. 
Table 3 (on next page)

Table 3. Primers sequences 


\begin{tabular}{|l|l|}
\hline $\begin{array}{l}\text { Mutagenic primers } \\
\text { G454V (\#10336022: P180917-006 A01 \& }\end{array}$ & $\begin{array}{l}\text { Fwd:CTGCGACGACATGGGAAAGTAGACTCTCCTGAG } \\
\text { GCCGT }\end{array}$ \\
& $\begin{array}{l}\text { Rev:ACGGGCCTCAGGAGAGTCTACTTTCCCATGTCG } \\
\text { TCGCAG }\end{array}$ \\
\hline $\begin{array}{l}\text { Primers with Kozak and restriction } \\
\text { enzymes to facilitate transference to the } \\
\text { eukaryotic plasmid }\end{array}$ & Fwd (Kpnl-Kozak-chVCL): \\
ch-VCL (\#10336022:379546 A01 \& A02) & RV (EcoRI-STOP-ch VCL): \\
\cline { 2 - 3 } & 5->3: AGCTgaattcctaCTGATACCATGGGGTC (29 nt) \\
\hline
\end{tabular}

1

2 Table 3. Primers sequences 


\section{Figure 1}

Cell shape index:design and proof of concept.

(A) Roundness, circularity and their quotients were initially measured and calculated for geometric figures. Here are Cshin values for some geometric figures and the correspondent figures with wavy limits. (B) NMuMG cells are a well-known model to study a process through which an epithelial cell loses its epithelial characteristics and acquires mesenchymal characteristics. Such process is an epithelial to mesenchymal transition (EMT) and is induced by treatment with TGF- $\beta$. We induced that process and measured Cshin to validate the proposed index

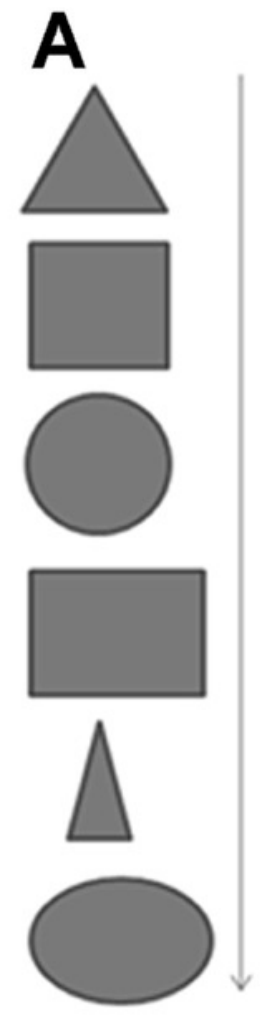

1,62

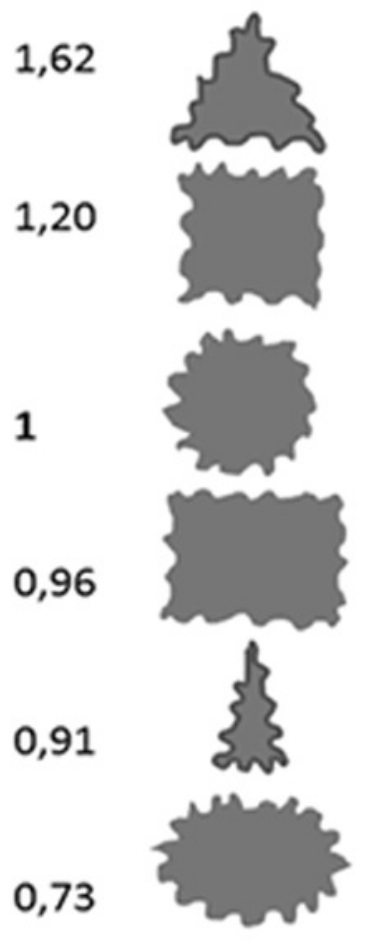

2,63

1,20

1,81

B

1,32

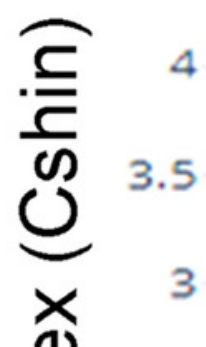

****

1,81

응 2.5

1,73

$\frac{\mathscr{E}}{\frac{1}{\sigma}}$

1,55

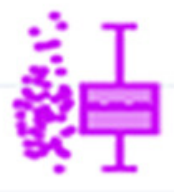

NMuMG

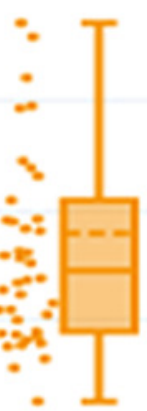

1.5
1
0.5 


\section{Figure 2}

VCL sequence harbors three TBMs conserved in vertebrates, one of which is present in Drosophila.

(A) TBMs, according to the hexamer rule for c-TBMs or the heptamer rule for nc-TBMs, follow the patterns: $R$ XXOX G or R XXXOX G, where $X$ is any aminoacid and $O$ is $G, P, A$ or $C$. These two patterns were searched and found conserved in vertebrates (Human, Mouse, Chicken, Xenopus and Zebrafish) as indicated under TBM-I, TBM-II and TBM-III. Only one of them was present in Drosophila while none of them was present in Caenorhabditis elegans. The single TBM present in Drosophila can align with human TBM-I or TBM-II depending on the matrix used for the alignment. (B) Superimposition of hVCL (blue, PDB:1TR2, B subunit) and dVCL predicted structure (grey). TBMs from hVCL are highlighted using the same color code than in panel A, while Drosophila TBM is colored in pink. Overlapped hVCL and Drosophila VCL structures evidence the equivalence of Drosophila TBM and vertebrate TBM-II. 


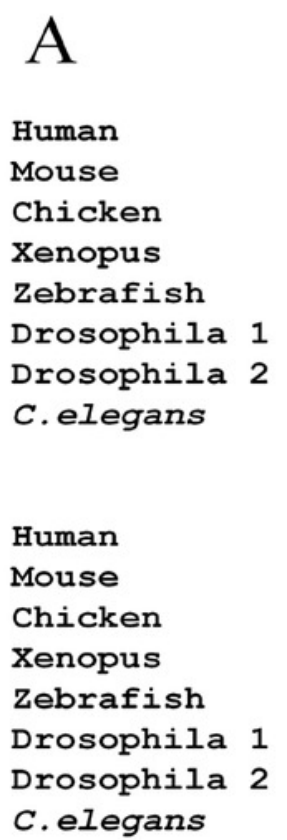

TBM-I

QILDEAGKVGELCAGKERREILGTCKMLGQMTDQVADLRARGQGSSPVAMQKAQQVSQGL 360 QILDEAGKVGELCAGKERREI LGTCKMLGQMT DQVADLRARGQGASPVAMQKAQQVSQGL 360 QILDEAGKAGELCAGKERREI LGTCKTLGQMT DQLADLRARGQGATPMAMQKAQQVSQGL 360 QILDEAGKVGELCAGTERKDI LGICRTLGQMTDQVSDLRARGQGATPIAMQKAQQVSQGL 360 QI LDEAGKVGELCAGKERREI LGTAKTLGQMTDQVSDVRARGQGATPMGMQKAQQVAQGL 361 QVIDNATEISERCLPQDSYPIRKLADEVTAMANTLCELRQEGKGQSP------------- 346 QVIDNATEISERCLP--------------------------QDSYPI------------ 320 RICEYADRISARALPEDAQSIKRS IFEITSFTDELCNLRNNGQP---DRENLAAQTARRL 357

TBM-II

LCDDPKERDDI LRSLGEISALTSKLADLRRQGKGDSPEARALAKQVATALQNLQTKTNRA 480 LCDDPKERDDILRSLGEIAALTSKLGDLRRQGKGDSPEARALAKQVATALQNLQTKTNRA 480 LCEEPKERDDI LRSLGEISALTAKLSDLRRHGKGDSPEARALAKQIATSLQNLQSKTNRA 480 LCEDPKDKEDI LRSLGEIAALTAKLTDLRRQGKGDSHEARALAKQIATSLQNLQTKVNRA 480 LCEDPKERDDI LRS IGEIAGLTARLVELRRIGKGDTPEARALAKQIGTALQNLQAKTNRA 480 LVRGIRDRMGELKSLVHQAVLGVDKAGVQQTAHT----------------------- 384 -------------EVTAMANT LCELRQEGKGQSPQAESLVRGIRDRMGELKSLVHQA 369

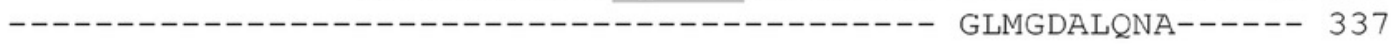

TBM-III

VANSRPA---KAAVHLEGKIEQAQRWIDNPTVDDRGVGQAAIRGLVAEGHRLANVMMGPY 537 VANSRPA---KAAVHLEGKIEQAQRWI DNPTVDDRGVGQAAIRGLVAEGHRLANVMMGPY 537 VANTRPV---KAAVHLEGKIEQAQRWIDNPTVDDRGVGQAAIRGLVAEGRRLANVMMGPY 537 VANSRPV---KAAVNMEGKVEQAQRWI DNPSVDDKGVGQAAIRGLVAEGRRLANSMIGPF 537 VANMRPA---KAAVTLEGKMEQALRWINNPGVDDHGVGQAAIRGLIAEGRRLASSLPGPY 537 --------------IQGRLEQAVKWLQHPEINDGGLGERAINLIVEEGRKVAEGCPGHQ 429 VLGVDKAGVQQTAHTIQGRLEQAVKWLQHPEINDGGLGERAINLIVEEGRKVAEGCPGHQ 429 ------A---NPAHTAAGRLEQALRWLDNPGLDDGGLGLQALRLLTADARKLADRLNPQD 433

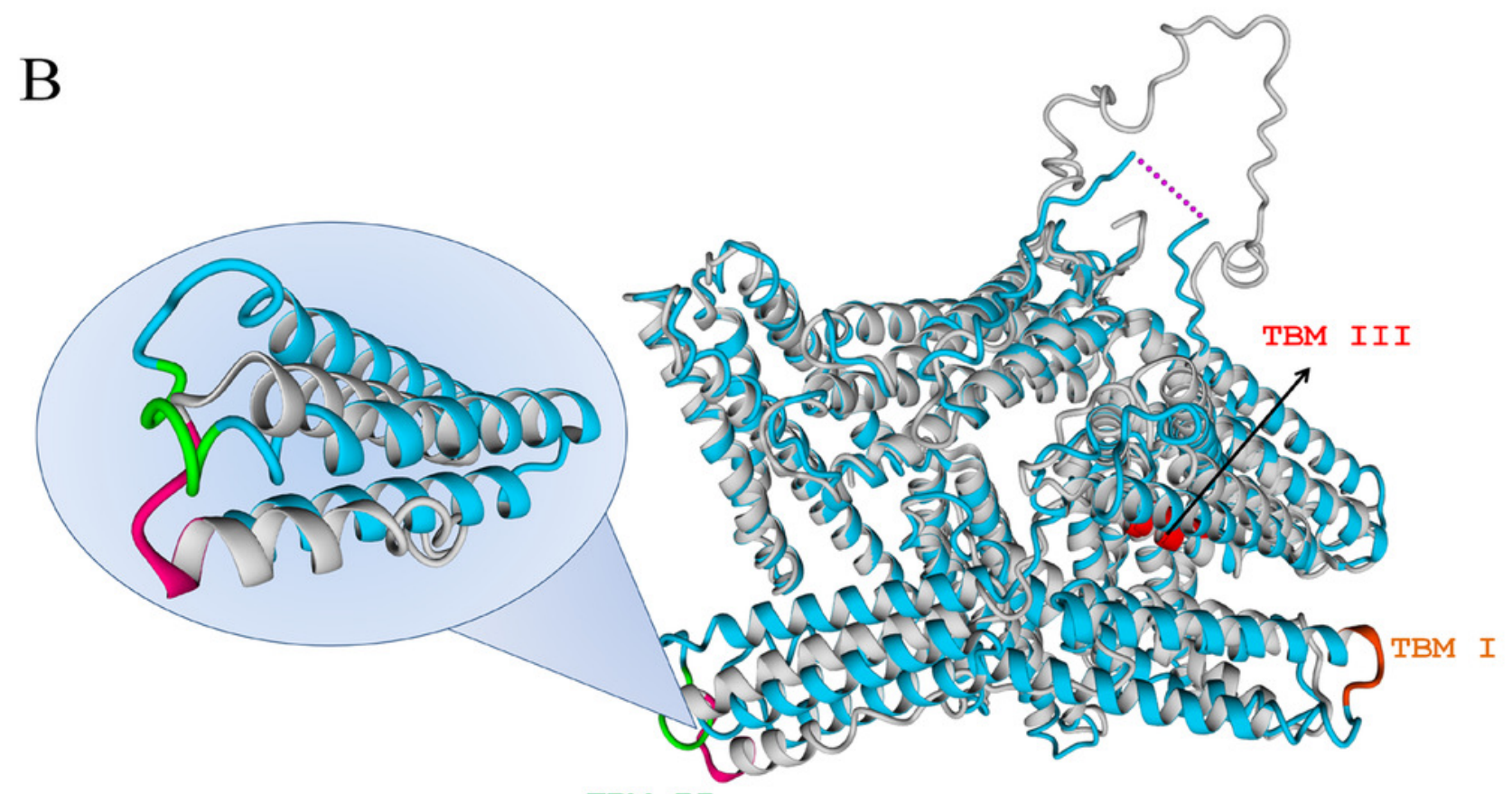

TBM II

Drosophila TBM 


\section{Figure 3}

Endogenous TNKS and PAR distribution in epithelial cells

(A) Confluent Vero cells were subjected to subcellular fractionation. The identity of the obtained fractions was confirmed using fractionation validation markers: for nuclear fraction (NF), Lamin A/C; for cytoplasmic fraction (CF), CALR3; for internal membrane fraction (IMF), GRASP; and for plasma membrane fraction (PMF), PMCA. TNKS was more abundant in IMF, but was also detected in the other cell fractions, including the PMF. PAR (detected with antiPAR reagent MABE-1031) was more abundant in the nuclear fraction but also detectable in the other cell fractions. Arrows on the right indicate discrete bands corresponding to unknown PARylated proteins observed in the PMF. (B-E) ICF to detect TNKS cellular localization (green), counterstained with F-actin (red) and DAPI (b/ue). A non-symmetric perinuclear region probably correspondent to the Golgi system harbored most TNKS. However, (D, E) a closer examination evidenced a wider TNKS distribution, including plasma membrane or submembrane regions, as indicated by white arrows. Bar: $25 \mu \mathrm{m}$. 

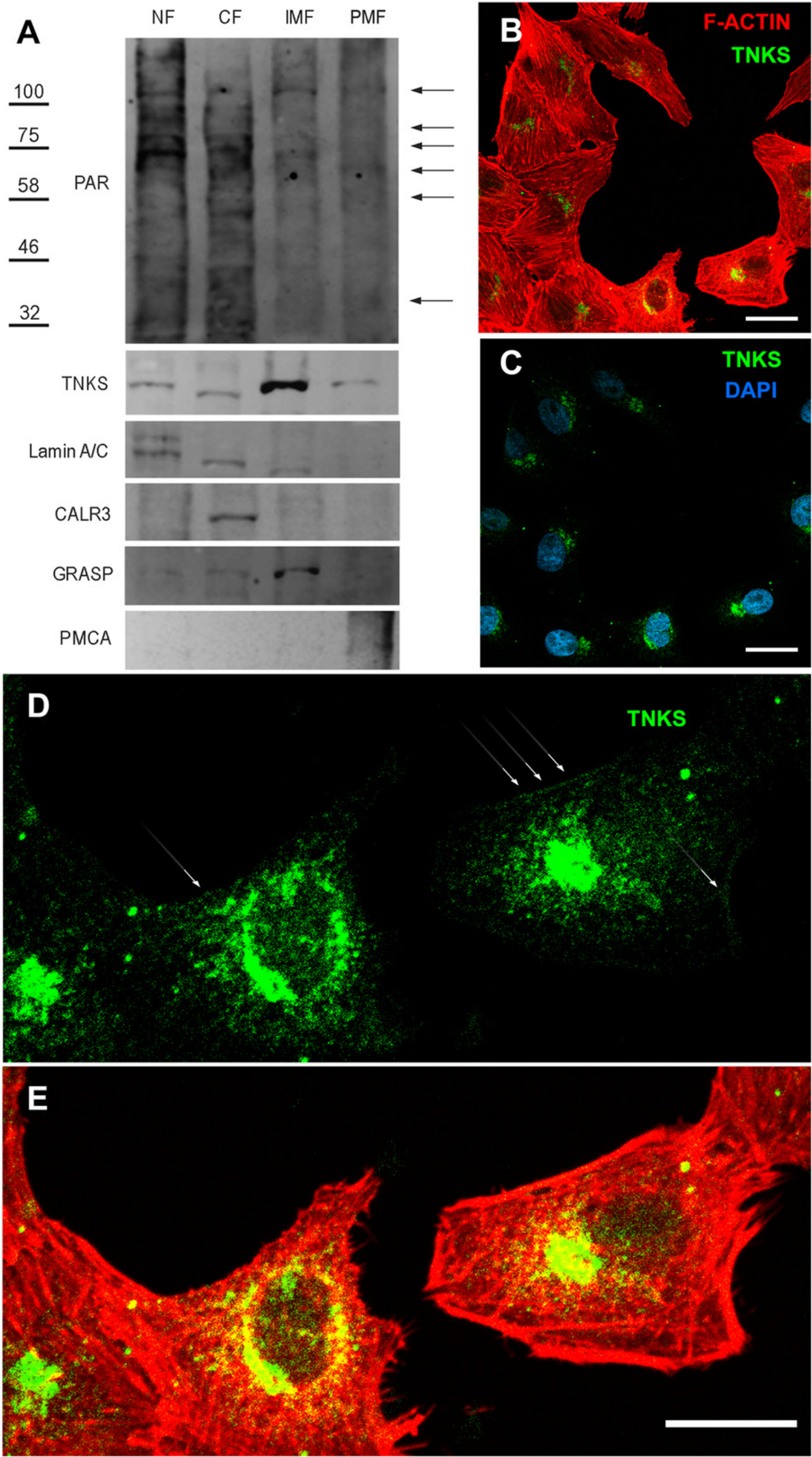

Peer) reviewing PDF | (2020:10:54544:2:0:CHECK 16 Apr 2021) 


\section{Figure 4}

TNKS inhibitors hampered PAR belt synthesis and altered the subcortical actin ring.

TNKSi were added to Vero cells at the moment of seeding and after $5 \mathrm{~h}$ cells were fixed, washed in PBS and subjected to ICF to detect PAR (green), F-actin with rhodamine-phalloidin counterstain (red) and nuclei with DAPI (blue). (A-E) PAR channel; (F-J) F-actin channel, (K-O) merged channels. (A, F, K) control, (B, G, L) 80 nM FLALL-9, (C, H, M) 60 nM MN-64, (D, I, N) $250 \mathrm{nM} \mathrm{G007-LK,} \mathrm{(E,} \mathrm{J,} \mathrm{O)} 2.6 \mu \mathrm{M}$ OD35. Instead of the tight straight continuous appearance of the belt $(\mathbf{A}, \mathbf{F}, \mathbf{K})$, a serrated discontinuous distribution was observed (B-O). Bar: $25 \mu \mathrm{m}$. For a quantitative expression of cell shape change, see Fig. 5. 


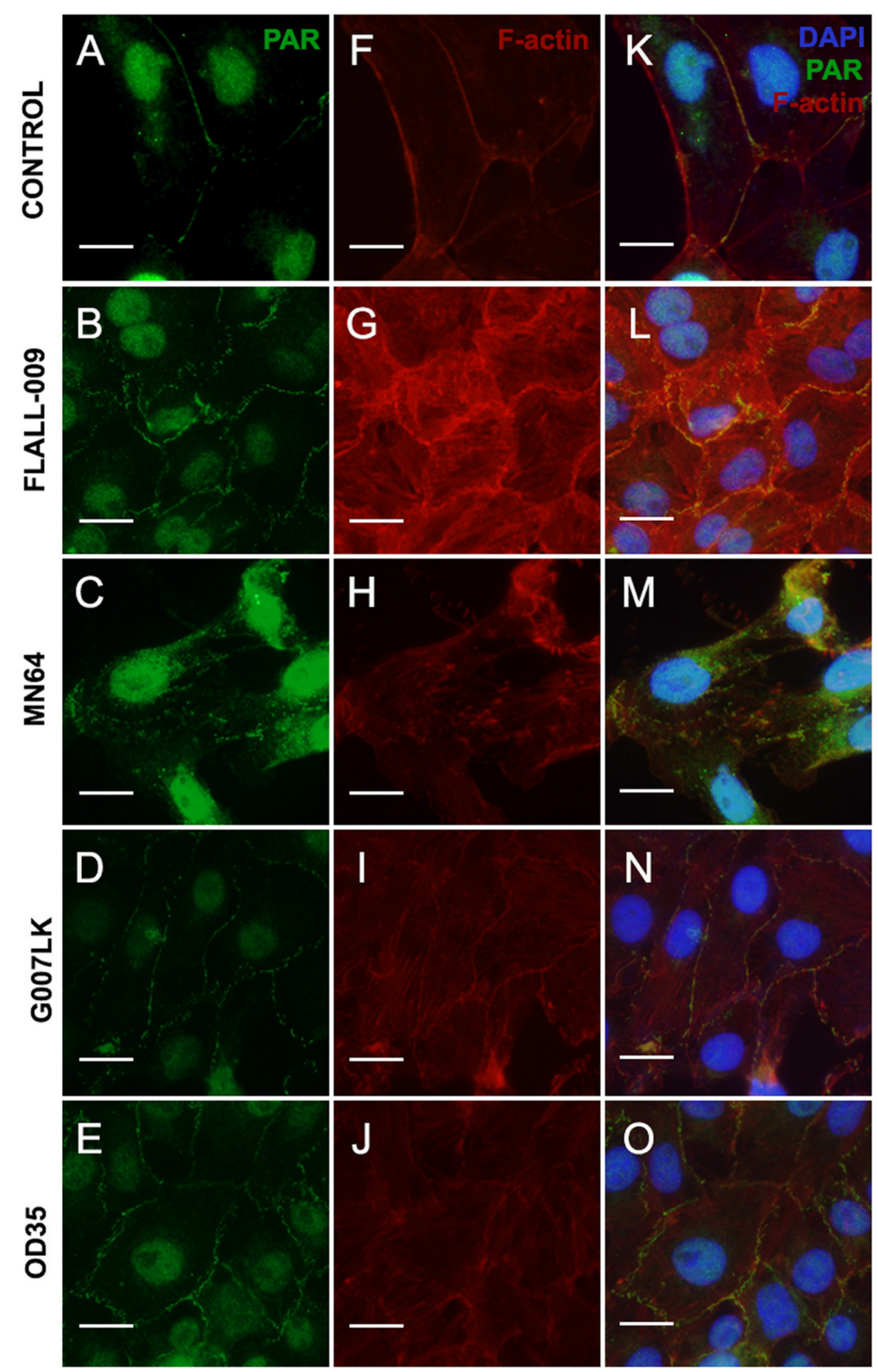

Peer) reviewing PDF | (2020:10:54544:2:0:CHECK 16 Apr 2021) 
Figure 5

TNKSi changed cell shape

Cells were exposed to the PARP-1/2-3 inhibitor Olaparib (OLA) or to TNKSi FLALL, MN64, G007LK or OD35, at the moment of seeding. Five hours later, they were fixed after a quick wash and subjected to ICF (see images in Fig. 4). Cell shape index was calculated as: CShin $=$ Roundness/Circularity. To appreciate the overall behavior of CShin and its validation as an ides, see Fig. 1.

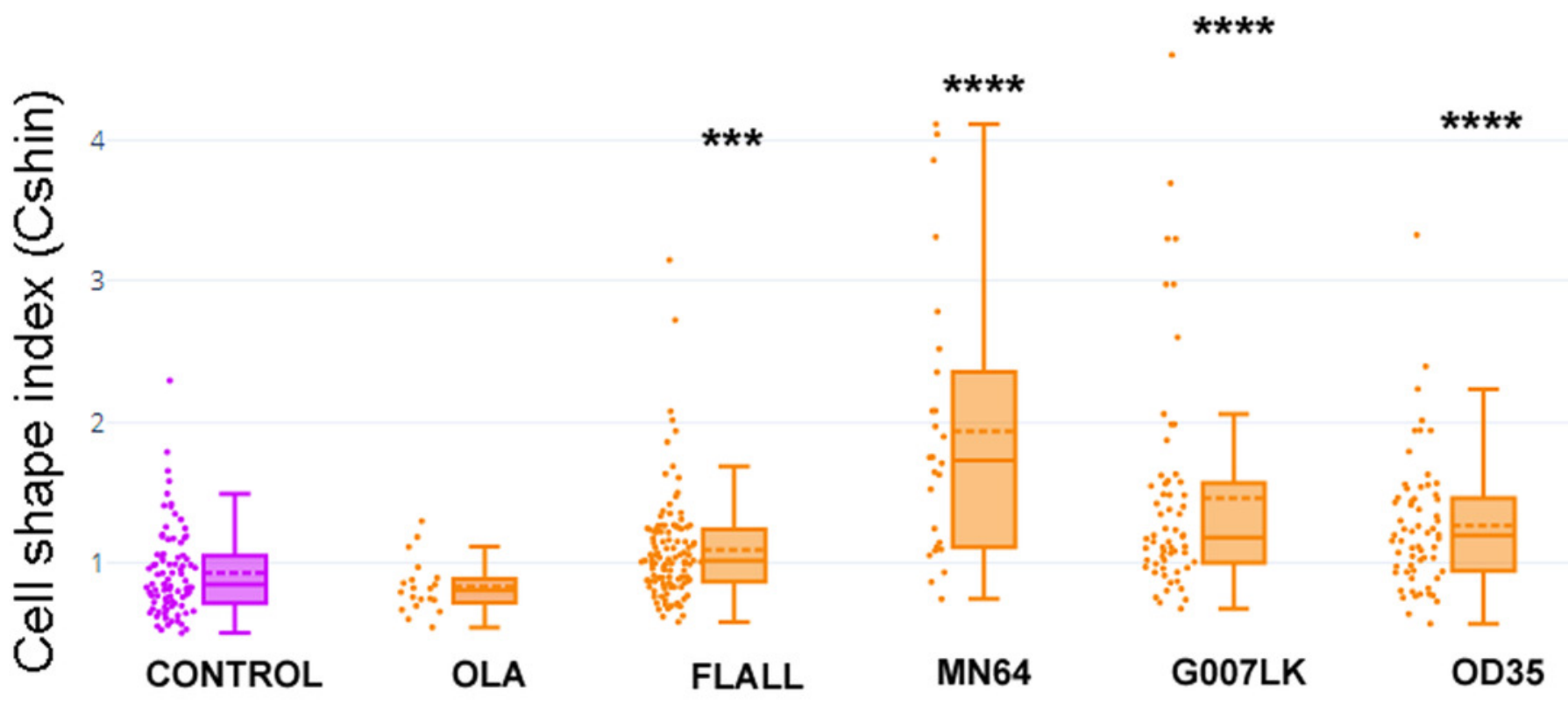




\section{Figure 6}

\section{A VCL pool was PARylated}

(A) Vero cell proteins were affinity precipitated using PAR-recognizing WWE-resin to recover covalently PARylated proteins. The flowthrough (FT) and affinity precipitated fractions (AP) were subjected to WB. Five cell junction and cytoskeletal proteins were assayed. Unlike $\beta$ catenin, E-cadherin, actin and $\alpha$-tubulin, a VCL pool was affinity precipitated (the arrow points to the lower apparent MW VCL band, around 90 to $100 \mathrm{Kda}$, which was the most abundant in the AP fraction). As expected, the resin did also precipitate PARylated PARP-1 and PAR itself. The species in which each antibody was raised is indicated under the correspondent label ( $R$ : rabbit; M: mouse). Except for mouse anti- $\alpha$-tubulin and mouse anti-VCL, all primary antibodies used (anti- $\beta$-catenin, anti-E-cadherin, anti-PARP-1, anti-PAR) were of rabbit origin or directly labeled (anti-actin). (B) Diagram indicative of the relative position of confocal images of the right panels (C-H): ICF anti-VCL (red) and anti-PAR (green), merged channels; (I - N) correspondent VCL and PAR masks; (O-T): in yellow, masks product highlighting the overlapping VCL and PAR regions, drawing precisely the epithelial belt. Subsequent confocal planes, from basal to apical, allowed the distinction of $\mathrm{VCL}$ in focal adhesions (eg. $\mathbf{C}, \mathbf{I}, \mathbf{0}$ ) from VCL associated to the PAR belt (eg. G, M, S). Bar: $25 \mu \mathrm{m}$. (U, V): VCL and PAR region of interest (ROI) around the belt (cropped from $\mathbf{E}$ ). (W): ++PDMs from an intensity correlation colocalization analysis. The pixels with values above the mean of each channel, in both channels, are depicted. (X): in yellow, the product of $\mathbf{U}$ and $\mathbf{V}$ masks (as in (O-T)). Notice that the complex intensity correlation analysis and masks images multiplication lead to the same result: colocalizing PAR and VCL pixels are on the epithelial belt. (Y): enlarged belt masks ROls extracted from (L-N), facilitate the precise visualization of colocalizing pixels. Overlapping percentages were calculated according to Mander's coefficients (small numbers or results section). 

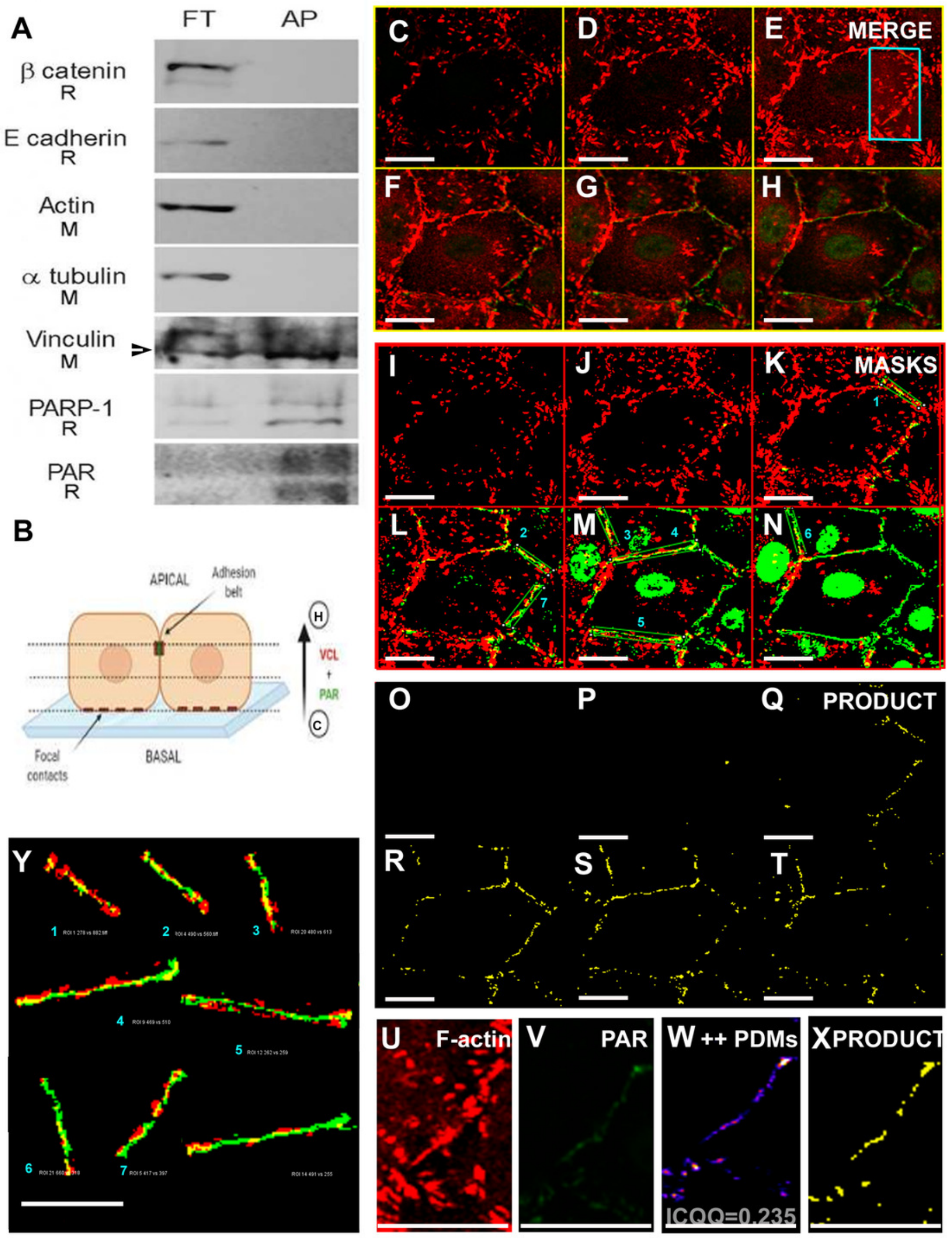


\section{Figure 7}

Transfection of MCF-7 (P10) monolayer with Tol2 14,5 KB eukaryotic expression vectors harboring VCL or VCL/*TBM.

(A) Circular PCR was carried on the $8909 \mathrm{pb}$ plasmid template $\mathrm{pET15b/GgVCL}$ using G454V mutagenic primers to generate $\mathrm{pET15b/GgVCL/*TBM} \mathrm{(B)} \mathrm{Schematic} \mathrm{representation} \mathrm{of} \mathrm{the}$ 14.5 KB eukaryotic expression plasmids Tol2/Gg-VCL and Tol2/Gg-VCL/*TBM, expressing the correspondent WT or mutated VCL under a $\beta$-actin promoter, plus green fluorescent protein whose expression relied on an Internal Ribosome Entry Site (IRES-GFP). In this way, transfected cells were expected to harbor free GFP (being easily identifiable) while expressing untagged VCL.

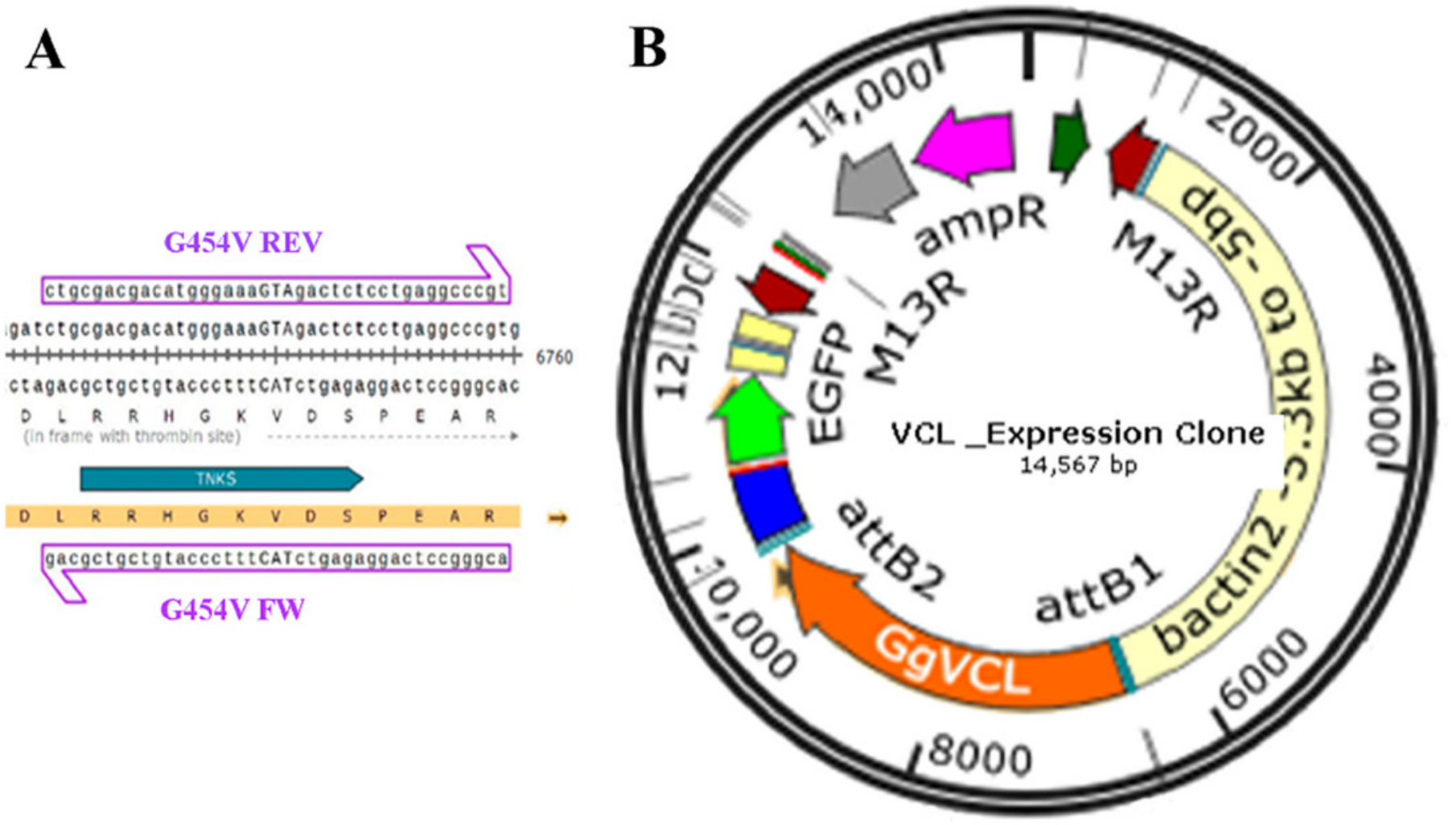




\section{Figure 8}

Transfection with Tol2-GgVCL or Tol2-GgVCL/*TBM

MCF-7 (P10) monolayers were transfected with either Tol2-GgVCL or Tol2-GgVCL/*TBM. Cells were fixed $17 \mathrm{~h}$ later. GFP (green) and VCL (red) were detected by ICF. The left half of the figure (A, B, E-J) corresponds to Tol2-GgVCL (WT); the right half (C, D, K-O) corresponds to Tol2-GgVCL/*TBM. (A-O): mixed channels; ( $\mathbf{A}^{\prime}-\mathbf{O}^{\prime}$ ): only GFP; (A' '-0"): only VCL. Notice the abundant VCL expression (neighbor cells in A are hardly detected) and the irregular, mesenchymal-like shape of cells harboring mutated VCL. Bar: $25 \mu \mathrm{m}$. 


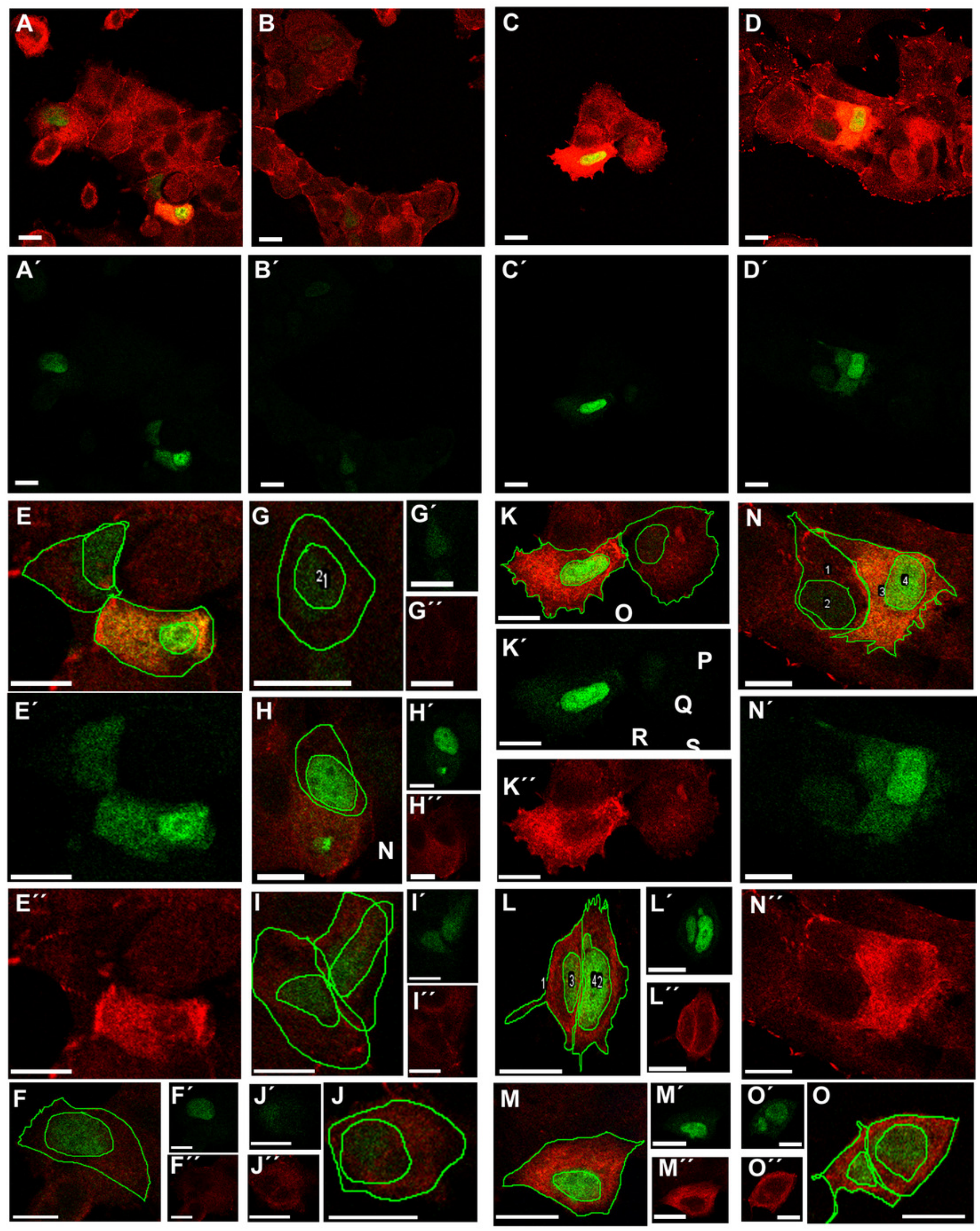


Figure 9

Figure 9. Cell shape was different in cells transfected with WT or TBM*-GgVCL.

Cell shape index after transfection with WT VCL was below 1 , as in other epithelial cells, while transfection with $\mathrm{VCL} /$ *TBM significantly increased the index value. 


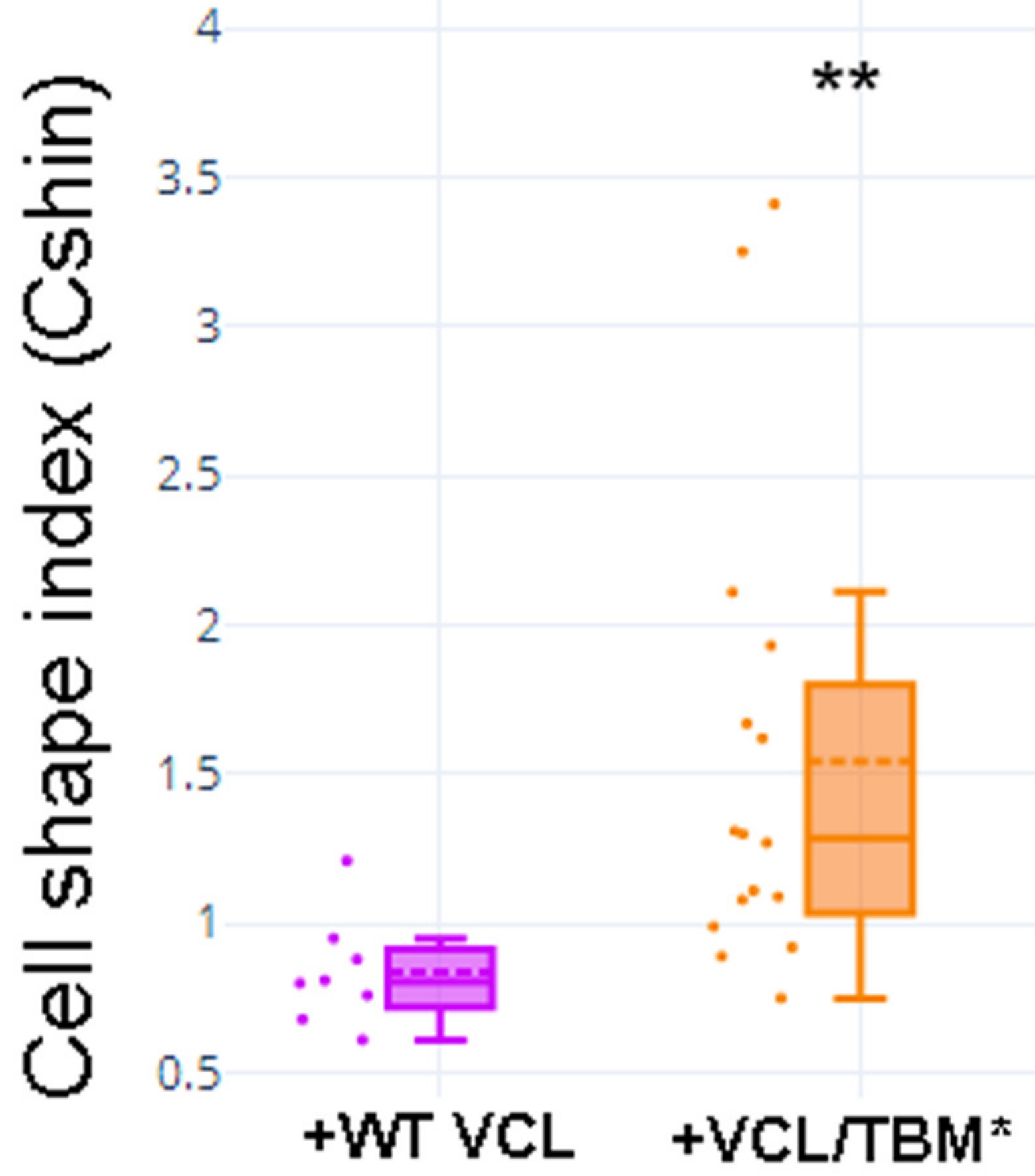

